\title{
DYNAMIC SOIL PRESSURES ON RIGID VERTICAL WALLS
}

\author{
A.S. Veletsos and A.H. Younan \\ Rice University, Houston, Texas
}

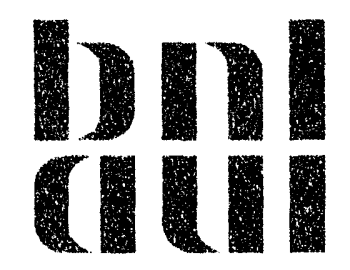

Under contract to

Brookhaven National Laboratory

Upton, New York 11973

December 1992

DEPARTMENT OF NUCLEAR ENERGY

BROOKHAVEN NATIONAL LABORATORY, ASSOCIATED UNIVERSITIES, INC.

UPTON, NEW YORK 11973

UNITED STATES DEPARTMENT OF ENERGY

CONTRACT NO. DE-ACO2-76CH0O016 
BNL 52357

UC-406

UC-510

BNL- -52357

DE93 008899

\title{
DYNAMIC SO!L PRESSURES ON RIGID VERTICAL WALLS
}

\author{
A.S. Veletsos and A.H. Younan \\ Rice University, Houston, Texas
}

Under contract to

Brookhaven National Laboratory

Upton, N.Y. 11973

December 1992

Prepared for

OFFICE OF ENVIRONMENTAL RESTORATION AND WASTE MANAGEMENT DEPARTMENT OF ENERGY, WASHINGTON, D.C.

This work was performed under the auspices of the U.S. Department of Energy by

Brookhaven National Laboratory under Contract Number DE-AC02-76-CH00016

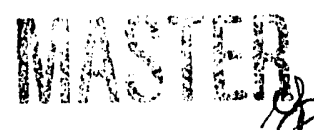

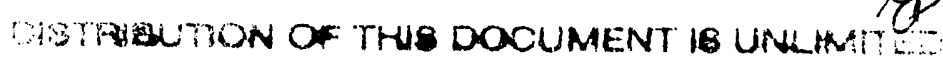




\section{DISCLAIMER}

This report was prepared as an account of work sponsored by an agency of the United States Government. Neither the United States Government nor any agency thereof, nor any of their employees, nor any of their contractors, subcontractors, or their employees, makes any warranty, express or implied, or assumes any legal liability or responsibility for the accuracy, completeness, or usefulness of any information, apparatus, product, or process disclosed, or represents that its use would not infringe privately owned rights. Reference herein to any specific commercial product, process, or service by trade name, trademark, manufacturer, or otherwise, does not necessarily constitute or imply its endorsement, recommendation, or favoring by the United States Government or any agency, contractor or subcontractor thereof. The views and opinions of authors expressed herein do not necessarily state or reflect those of the United States Government or any agency, contracto: or subcontractor thereof.

Printed in the United States of America Available from

National Technical Information Service

U.S. Department of Commerce

5285 Port Royal Road

Springfield, VA 22161

NTIS price codes:

Printed Copy: A04; Microfiche Copy: A01 


\begin{abstract}
A critical evaluation is made of the dynamic pressures and the associated forces induced by ground shaking on a rigid, straight, vertical wall retaining a semi-infinite, uniform viscoelastic layer of constant thickness. The effects of both harmonic and earthquakeinduced excitations arc examined. Simple approximate expressions for the responses of the system are developed, and comprehensive numerical data are presented which elucidate the effects and relative importance of the various parameters involved. These solutions are then compared with those obtained by use of a simple model proposed previously by Scott, and the accuracy of this model is assessed. Finally, two versions of an alternative model are proposed which better approximate the action of the system. In the first, the properties of the model are defined by frequency-dependent parameters, whereas in the second, which is particularly helpful in analyses of transient response, they are represented by frequency-independent, constant parameters.
\end{abstract}




\section{TABLE OF CONTENTS}

Section Page

1 INTRODUCTION 1-1

2 SYSTEM AND METHOD OF ANALYSIS $2-1$

2.1 Governing Equations and Assumptions $2-1$

2.2 Harmonic Response $2-4$

$\begin{array}{ll}\text { 2.2.1 Vertical Displacements } & 2-7\end{array}$

2.2.2 Normal and Shearing Stresses $\quad 2-7$

2.2.3 Wall Pressures and Forces $\quad 2-8$

2.3 Transient Response 2-9

3 PRESENTATION AND ANALYSIS OF RESULTS 3-1

3.1 Static Effects 3-1

3.2 Results For Harmonic Response 3-2

3.2.1 Magnitude and Distribution of Wall Pressures 3-2

3.2.2 Horizontal Variation of Effects $3-3$

3.2.3 Base Shear and Moment $3-4$

3.3 Solutions for Transient Response 3-5

4 MODELING OF SYSTEM 4-1

4.1 Scott's Model 4-1

4.2 Proposed Models 4-3

4.2.1 Model with Constant Parameters 4-3

4.2.2 Model with Frequency-Dependent Parameters

4.2.3 Model with Modified Frequency-Independent Parameters 4-6

5 CONCLUSIONS 5-1

6 REFERENCES 6-1

7 NOTATION $7-1$

8 APPENDIX 8-1

8.1 Harmonic Response of Uniform Shear-Beam 8-1 


\section{LIST OF FIGURES}

Figure $\quad$ Page

$\begin{array}{lll}2.1 & \text { System considered. } & 2-10\end{array}$

3.1 Comparison of static values of base shear in wall computed by different procedures. 3-7

3.2 Frequency response plots for coefficients in expression for amplitude of pressure induced at top of wall by harmonic input motion; $\delta=0.1$. $3-8$

3.3 Heightwise variations of coefficients in expression for wall pressure induced by harmonic input motion; $\delta=0.1$.

3.4 Horizontal variations of horizontal normal stresses induced at top of layer by harmonic base motion; $v=0.3, \delta=0.1$.

3.5 Horizontal variations of top displacements and base shearing stresses induced by harmonic base motions; $v=0.3, \delta=0.1$.

3.6 Effect of soil material damping on amplitudes of base shear induced in wall of harmonically excited systems; $v=0.3$.

3.7 Comparison of frequency response curves for base shear in wall computed by considering one and all modes of vibration; $v=0.3, \delta=0.1$.

3.8 Height of resultant of total wall force induced by harmonic base excitation; $v=0.3$. 3-14

3.9 Comparison of maximum base wall-shears computed for El Centro record from analytical solution and Scott's model; $v=0.3, \delta=0.1$.

4.1 Scott's model.

4.2 Frequency response curves for real and imaginary parts of pressures computed for top of wall by analytical solution and Scott's model; $v=0.3, \delta=0.1$.

4.3 Comparison of frequency response curves for base wall-shears computed by analytical solution and Scott's model; $v=0.3, \delta=0.1$.

4.4 Proposed model.

4.5 Comparison of frequency response curves for base wall-shears computed from analytical solution and model with frequency-independent parameters; $v=0.3, \delta=0.1$. 


\section{LIST OF FIGURES (Continued)}

4.6 Factors in expressions for dynamic stiffness and damping coefficients of proposed model with frequency-dependent parameters; $v=0.3$.

4.7 Response spectra for base wall-shears computed for El Centro record from analytical solution and proposed model with frequency-independent parameters; $v=0.3$.

4-13 


\section{EXECUTIVE SUMMARY}

The study reported herein represents the first step in a broader investigation into the response to ground shaking of embedded and underground structures. It presents a comprehensive evaluation of the dynamic pressures and the associated forces induced by ground shaking on a rigid, straight, vertical wall retaining a semi.infinite, uniform viscoelastic layer of constant thickness. The effects of both harmonic and earthquake-induced excitations are examined. Simple approximate expressions for the responses of the system are developed, and comprehensive numerical data are presented which elucidate the effects and relative importance of the various parameters involved. These solutions are then compared with those obtained by use of a simple model proposed previously by Scott, and the accuracy of this model is assessed. Finally, two versions of an alternative model are proposed which better approximate the action of the system. In the first, the properties of the model are defined by frequency-dependent parameters, whereas in the second, which is particularly helpful in analyses of transient response, they are represented by frequency-independent, constant parameters.

With the analytical expressions presented, the dynamic pressures and the associated forces exerted on the wall may be evaluated readily and reliably. Additionally, the numerical data presented and the analysis of these data provide valuable conceptual frameworks for the interpretation of solutions for more involved systems as well.

Scott's model, which ignores the radiational damping capacity of the medium and considers the wall pressures to be proportional to the relative motions of the wall and the soil at the far field, does not adequately describe the action of the system and may lead to large errors. The proposed alternative models, which effectively consider the wall pressures to be governed by the action of the soil in the immediate vicinity of the wall, better characterize the response of the system, and lead to results of reasonable accuracy. In particular, the model with frequency-independent parametcrs may be used to evaluate readily and reliably the effects of earthquake ground motions of broad-banded spectral characteristics. 


\section{ACKNOWLEDGMENT}

This study was carried out at Rice University under Grant 568821 from the Brookhaven National Laboratory, Upton, New York. This support is acknowledged gratefully. Appreciation also is expressed to Drs. M. Reich and K. Bandyopadhyay for their understanding project management. 


\section{SECTION 1}

\section{INTRODUCTION}

The evaluation of the dynamic soil pressures induced by ground shaking on vertical walls is fundamental to the seismic analysis and design of earth retaining structures and of embedded and underground systems. Although these systems have been the subject of numerous studies over the years, their dynamic response is still not well understood. There is, in particular, lack of adequate response data and of rational but simple methods of analysis which may be used reliably and cost-effectively in design. Valuable accounts of previous contributions on the subject have been provided in state-of-theart reports by Nazarian and Hadjian [1], Prakash [2] and Whitman [3], and they will not be highlighted here.

The methods that have been used for the analysis of the problem can conveniently be classified into three categories: (1) those in which the relative motions of the wall and backfill material are sufficiently large to induce a limit or failure-state in the soil; (2) those in which the wall is essentially unyielding and the ground motion is of sufficiently low intensity so that the backfill material may be considered to respond witliin the linearly elastic range of deformations: and (3) the intermediate case in which the true nonlinear, hysteretic properties of the soil are accounted for explicitly.

Representative of the first approach is the well known Mononobe-Okabe method $[4,5]$ and its various variants $[6,7,8]$, in which a wedge of soil bounded by the wall and an assumed failure plane is considered to move as a rigid body, experiencing the same acceleration as the ground. Representative of the third approach is a recent contribution by Siller et al [9] dealing with the responses of gravity and anchored walls.

The present study is in the vein of the second approach; it examines the wall pressures and associated forces induced by ground shaking under elastic conditions of response. The sy',tem investigated is a semi-infinite, uniform, viscoelastic stratum of constant thickness which is excited by a space-invariant motion along its base and its vertical boundary. Boih harmonic and transient excitations are used. Relatively simple, approximate solutions and comprehensive numerical data are presented which elucidate the effects and relative importance of the numerous parameters involved. In addition to valuable insights into the response of the particular system considered, the numerical solutions presented provide a convenient frame of reference for the analysis and interpretation of the responses of more com- 
plex systems as well.

The elastic response of this system has been examined previously by Matuo and Ohara [10], who employed a method of analysis similar to the one used here. However, the accuracy of their solution cannot be confirmed, and they have presented no numerical solutions. In a series of valuable studies $[11,12]$, Wcod has provided analytical solutions and comprehensive numerical data for the response of a stratum of finite length excited uniformly along its base and its two vertical boundaries. Wood's analysis was implemented by modal superposition and led to expressions involving double series. While these expressions can also be used to approximate the response of the semi-infinite stratum considered herein, they require the consideration of a prohibitively large number of terms.

Scott [13] has proposed a simple model for approximating the responses both of the semi-infinite stratum examined here and of the bounded system investigated by Wood [11, 12]. In this model, the far field response of the stratum is evaluated from an analysis of a similarly excited, uniform shear-beam. The dynamic wall pressure at an arbitrary height is then computed on the assumption that it is proportional to the relative motions of the wall and the shear-beam at that height. Scott's model has been used widely [14 through 18] and variations of it have also been employed in analyses of piles [19] and of underground cylindrical structures [20].

One of the objectives of the present study is to assess the reliability of Scott's model for the problem under consideration by comparing its predictions with those of the more nearly exact analysis presented herein. A final objective is to present an alternative model which better approximates the action of the system. 


\section{SECTION 2}

\section{SYSTEM AND METHOD OF ANALYSIS}

The system investigated is shown in part (a) of Fig. 2:1. It is a semi-infinite, uniform layer of viscoelastic material that is free at the upper surface, is bonded to a rigid base, and is retained along one of its vertical boundaries by a rigid wall. Both the wall and the base of the layer are presumed to be excited by a space-invariant motion, the acceleration of which at any time $t$ is $\ddot{x}_{g}(t)$. Material damping for the medium is considered to be of the constant hysteretic type [21].

The properties of the layer are defined by its mass density, $\rho$, shear modulus of elasticity, G, Poisson's ratio, $v$, and the material damping factor, $\delta$, which is considered to be frequency-independent and the same for both shearing and axial deform ions. The latter factor is the same as the $\tan \delta$ factor used by Veletsos et al in several previous studi ... ondation dynamics (e.g., Refs. 21 and 22) and twice as large as the percentage of critical damping, $\beta$. used by other authors in related studies (e.g., Refs. $11,12,13$ and 23 ).

The displacements relative to the moving boundary and the resulting wall pressures and forces for the base-excited system can be shown to be identical to those of the force-excited system indicated in part (b) of Fig. 2.1. Both ihe wall and base of the latter system are stationary, and the layer is excited by uniform lateral body forces of intensity $-\rho \ddot{x}_{g}(t)$. For exciting frequencies that are very low in comparison to the fundamental natural frequency of the stratum, the action of the force-excited system may be easier to visualize than that of the base-excited system.

\subsection{Governing Equations and Assumptions}

When referred to the rectangular system of coordinates $x, y$ shown in Fig. 2.1, the equations of motion for the medium may be written as

$$
\begin{aligned}
& \frac{\partial \sigma_{x}}{\partial \xi}+\frac{\partial \tau_{x y}}{\partial \eta}=\rho H \frac{\partial^{2} u}{\partial t^{2}}+\rho H \ddot{x}_{g}(t) \\
& \partial \sigma_{y} \\
& \frac{\partial \eta}{\partial \eta}+\frac{\partial y}{\partial \xi}=\rho H \frac{\partial^{2} v}{\partial t^{2}}
\end{aligned}
$$

in which $\sigma_{x}$ and $\sigma_{y}$ are the normal stresses at an arbitrary point and time along the $x$ - and $y$-coordi- 
nates, respectively; $\mathrm{u}$ and $\mathrm{v}$ are the corresponding isplacements relative to the moving boundary: $\tau_{x y}$ is the shearing stress in the $x-y$ plane: a dot superscript denotes a differentiation with respect to time: and $\xi$ and $\eta$ are dimensionless position coordinates defined by $\xi=x / H$ and $\eta=y / H$. The sign convention for stresses and displacements is that used in theory of elasticity. Specifically, displacements are positive when directed along the positive direction of the corresponding coordinate axes, normal stresses are positive when they induce tension, and the positive directions of the shearing stresses are as indicated by the inset diagram in Fig. 2.1(a).

For the two-dimensional, plane strain condition examined herein, the stress components are related to the displacement components by

$$
\begin{aligned}
& \sigma_{x}=\left(\lambda^{*}+2 G^{*}\right) \frac{1}{H} \frac{\partial u}{\partial \xi}+\lambda^{*} \frac{1}{H} \frac{\partial v}{\partial \eta} \\
& \tau_{x y}=G^{*} \frac{1}{H}\left(\frac{\partial u}{\partial \eta}+\frac{\partial v}{\partial \xi}\right) \\
& \sigma_{y}=\left(\lambda^{*}+2 \cdot G^{*}\right) \frac{1}{H} \frac{\partial v}{\partial \eta}+\lambda^{*} \frac{1}{H} \frac{\partial u}{\partial \xi}
\end{aligned}
$$

in which $\mathrm{G}^{*}$ and $\lambda^{*}$ are the complex-valued Lame constants

$$
\begin{aligned}
& G^{*}=G(1+i \delta) \\
& \lambda^{*}=\frac{2 v}{1-2 v} G^{*}
\end{aligned}
$$

and $i=\sqrt{-1}$.

Fundamental to the analysis presented is the assumption that no vertical normal stresses develop anywhere in the medium. As a consequence, the following expression is obtained from Eq. 5

$$
\frac{\partial v}{\partial \eta}=-\frac{\lambda^{*}}{\lambda^{*}+2 G^{*}} \frac{\partial u}{\partial \xi}=-\frac{v}{1-v} \frac{\partial u}{\partial \xi}
$$

which when substituted into Eq. 3 leads to

$$
\sigma_{\mathrm{x}}=\Psi_{\mathrm{o}}^{2} \mathrm{G}^{*} \frac{1}{\mathrm{H}} \frac{\partial \mathrm{u}}{\partial \xi}
$$

where

$$
\Psi_{0}=\sqrt{\frac{2}{1-v}}
$$

Next, on substituting Eq. 3 along with Eq. 4 into Eq. 1, and making use of Eqs. 7 and 8, the equation expressing the equilibrium of forces in the $x$-direction can be written as 


$$
\Psi_{e}^{2} G^{*} \frac{\partial^{2} u}{\partial \xi^{2}}+G^{*} \frac{\partial^{2} u}{\partial \eta^{2}} \cdots H^{2} \frac{\partial^{2} u}{\partial t^{2}}=\rho H^{2} \ddot{x}_{g}(t)
$$

in which

$$
\Psi_{e}=\sqrt{\frac{2-v}{1-v}}
$$

Eq. 11 is solved subject to the boundary conditions

$$
\begin{aligned}
& \left.u\right|_{\xi=0}=0 \\
& \left.u\right|_{\eta=0}=0
\end{aligned}
$$

With u determined, the normal stresses are computed from Eq. 9; the vertical displacements, v, are determined by integration of Eq. 8 subject to the boundary condition

$$
\left.v\right|_{\eta=0}=0
$$

and the shearing stresses, $\tau_{x y}$, are determined from Eq. 4 .

It s'nould be noted that Eq. 2, which expresses the equilibrium of the vertical forces, is not satisfied in this approach, neither is the condition of vanishing shears at the free surface. For the horizontal ground motion being examined, the primary motions in the medium are shearing and longitudinal. The vertical motions and stresses are Poisson's ratio induced, and would not be expected to affect importantly the resulting wall pressures and forces which are the quantities of primary interest in this study. This expectation as well as the unimportance of violating the condition of zero shearing stresses at the surface are confirmed in a later section through studies of the static response of the system.

Note should also be taken of the fact that no mention has so far been made of the in-plane condition at the wall-soil interface. For the analysis presented, both the shearing stresses and the vertical displacements along the interface are generally finite. Accordingly, the presumed interface condition is neither smooth nor rough but intermediate in nature. This approximation is also examined further later.

If instead of the vertical normal stresses, $\sigma_{y}$, the vertical displacements, $v$, had been assumed to be zero, it can be shown that both the factor $\psi_{\mathrm{o}}$ in Eq. 9 and the factor $\psi_{\mathrm{e}}$ in Eq. 11 would have had to be replaced by

$$
\chi_{0}=\sqrt{\frac{2(1-v)}{1-2 v}}
$$

The assumption of vanishing vertical displacements underlies both the analysis presented by Matuo and Ohara [10] and the study of several related three-dimensional problems reported by Tajimi [23]. 
The interrelationship of the solutions based on the $\sigma_{y}=0$ and $v=0$ approximations is examined in a later section. In the interim, it is worth noting from Eqs. 16 and 9 that, as $v \rightarrow 0.5$, the longitudinal normal stresses in the solution for the $v=0$ approximation tend to infinity, a result which is clearly unacceptable. Except where specifically otherwise indicated, the solutions presented herein are for the assumption of vanishing vertical normal stresses.

\subsection{Harmonic Response}

For a harmonic input motion of acceleration

$$
\ddot{x}_{g}(t)=\ddot{x}_{g} e^{i \omega t}
$$

the relative displacements $u(\xi, \eta, t)$ may be expressed in the form

$$
u(\xi, \eta, t)=U(\xi, \eta) e^{i \omega t}
$$

in which $\ddot{X}_{\mathrm{g}}=$ the acceleration amplitude of the input motion; $\omega=$ the circular frequency of the excitation and resulting response: and $U(\xi, \eta)=$ a complex-valued function of $\xi$ and $\eta$ representing the relative displacement amplitudes.

The function $U(\xi, \eta)$ may be expressed as a linear combination of the natural modes of vibration of the medium computed on the assumption that it acts as a series of vertical cantilever shear-beams, i.e., as

$$
U(\xi, \eta)=\sum_{n=1,3}^{\infty} U_{n}(\xi) \sin \frac{n \pi}{2} \eta
$$

in which $\mathrm{n}=$ an odd integer. Note that in addition to satisfying the boundary condition defined by Eq. 14 , this expansion defines exactly the far field effects.

On substituting Eqs. 17 and 18 into Eq. 11, making use of Eq. 19, and expanding $\ddot{X}_{\mathrm{g}}$ in the form

$$
\ddot{X}_{g}=\frac{4}{\pi} \ddot{X}_{g} \sum_{n=1,3}^{\infty} \frac{1}{n} \sin \frac{n \pi}{2} \eta
$$

it is found that $U_{n}$ must satisfy the ordinary differential equation

$$
\frac{d^{2} U_{n}}{d \xi^{2}}-\left[\frac{n \pi}{2 \psi_{e}}\right]^{2} \Lambda_{n} U_{n}=\frac{4 \rho \ddot{X}_{g} H^{2}}{n \pi \Psi_{e}^{2} G^{*}}
$$

in which

$$
\Lambda_{n}=1-\frac{\phi_{n}^{2}}{1+i \delta}
$$




$$
\phi_{\mathrm{n}}=\frac{1}{\mathrm{n}} \frac{\omega}{\omega_{1}}
$$

$\omega_{1}=$ the fundamental circular natural frequency of the cantilever shear-beam, given by

$$
\omega_{1}=\frac{\pi v_{s}}{2} \frac{H}{H}
$$

and $v_{s}=\sqrt{G / p}=$ the shear wave velocity for the medium.

The homogeneous solution of Eq. 21, $U_{n}^{h}$, is given by

$$
U_{n}^{h}(\xi)=A_{n} e^{-\alpha_{n} \xi}+B_{n} e^{\alpha_{n} \xi}
$$

in which $A_{n}$ and $B_{n}$ are integration constants, and $\alpha_{n}$ is the square root of the coefficient of $U_{n}$ in Eq. 21. The latter quantity may be expressed as

$$
\alpha_{n}=\frac{n \pi}{2 \psi_{e}}\left(a_{n}+i b_{n}\right)=c_{n}+i d_{n}
$$

where $a_{n}$ and $b_{n}$ are real-valued positive dimensionless factors defined by

$$
\begin{aligned}
& a_{n}=\sqrt{\left|\Lambda_{n}\right|} \cos \theta_{n} \\
& b_{n}=\sqrt{\left|\Lambda_{n}\right|} \sin \theta_{n}
\end{aligned}
$$

and $\theta_{n}=$ a phase angle in thi range between 0 and $\pi / 2$ given by

$$
\theta_{n}=\frac{1}{2} \operatorname{Arg} \Lambda_{n}=\frac{1}{2} \arctan \frac{\delta \phi_{n}^{2}}{1-\phi_{n}^{2}+\delta^{2}}
$$

The vertical bars in the expressions for $a_{n}$ and $b_{n}$ represent the modulus of the enclosed quantity. For a non-dissipative, undamped medium, $\theta_{n}$ equals zero for $\phi_{n}<1$ and $\pi / 2$ for $\phi_{n}>1$ : accordingly, for both cases, $a_{n}+i b_{n}=\sqrt{1-\phi_{n}^{2}}$.

Considering that the term at the extreme right of Eq. 25 increases without bound with increasing $\xi$ while the response is finite, the integration constant $B_{n}$ must vanish. This reasoning is strictly valid only for a dissipative medium with a finite value of $\delta$. For a purely elastic medium and values of $\phi_{n}>1, \alpha_{n}$ is purely imaginary, and the term involving the factor $B_{n}$ does not increase unboundedly. Rather, its product with the time function $\mathrm{e}^{i \omega t}$ represents a harmonic wave propagating from the far field towards the wall. However, since no waves originate or are reflected from the far field. $B_{n}$ must again vanish.

The particular solution of Eq. $21, U_{n}^{p}$, is a constant given by 


$$
U_{n}^{p}(\xi)=-\frac{16 \rho}{\pi^{3}} \frac{\ddot{X}_{g} H^{2}}{G} \frac{1}{n^{3}} \cdot \frac{1}{1-\phi_{n}^{2}+i \delta}
$$

and the complete solution for $U_{n}(\xi)$ is given by

$$
U_{n}(\xi)=A_{n} e^{-\left(c_{n}+i d_{n}\right) \xi}-\frac{16}{\pi^{3}} \frac{\rho \ddot{X}_{g} H^{2}}{G} \frac{1}{n^{3}} \frac{1}{1-\phi_{n}^{2}+i \delta}
$$

in which the integration constant $A_{n}$ may now be determined from the boundary condition defined by Eq. 13.

On so determining $A_{n}$, and substituting the resulting expression for $U_{n}$ into Eq. 19, the following expression is obtained for the relative displacement amplitude

$$
U(\xi, \eta)=-\frac{16}{\pi^{3}} \frac{\rho \ddot{X}_{g} H^{2}}{G} \sum_{n=1,3}^{\infty} \frac{1}{n^{3}} \frac{1-e^{-\left(c_{n}+i d_{n}\right) \xi}}{1-\phi_{n}^{2}+i \delta} \sin \frac{n \pi}{2} \eta
$$

The real part of this equation represents a response component that is in phase with the exciting motion, whereas the imaginary part represents a component that is $90^{\circ}$ out of phase. Two factors contribute to the imaginary part: (1) the soil material damping: and (2) the radiational damping, which is associated with the capacity of the medium to dissipate energy by radiation of waves into the far field. The relative importance of these two sources of energy dissipation depends on the frequency of the excitation, and this dependen. $: x$ amined further in a later section.

Provided soil material damping is finite, as $\xi \rightarrow \infty$ the exponential term in Eq. 31 vanishes, and the equation reduces to

$$
U(\eta)=-\frac{16}{\pi^{3}} \frac{\rho \ddot{X}_{g} H^{2}}{G} \sum_{n=1,3}^{\infty} \frac{1}{n^{3}} \frac{1}{1-\phi_{n}^{2}+i \delta} \sin \frac{n \pi}{2} \eta
$$

This expression is, of course, the same as that governing the motion of a base-excited, vertical shearbeam of the same height and material properties as the stratum; see Appendix.

For a purely elastic medium with no material damping, Eq. 31 reduces to

$$
U(\xi, \eta)=-\frac{16}{\pi^{3}} \frac{\rho \ddot{X}_{\mathrm{g}} H^{2}}{G} \sum_{n=1,3}^{\infty} \frac{1}{n^{3}} \frac{1-e^{-\frac{n \pi}{2 \psi_{e}} \sqrt{1-\phi_{11}^{2}} \xi}}{1-\phi_{n}^{2}} \sin \frac{n \pi}{2} \eta
$$

The following trends are worth noting in this expression:

1. For exciting frequencies lower than the fundamental natural frequency of the layer (values of $\left.\phi_{1}<1\right)$, the exponential functions for all terms in the series are real-valued, and the displacement 
amplitudes increase monotonically from zero at $\xi=0$ to their maximum values at the far field: the latter values are the same as those given by the undamped version of Eq. 32. The lack of any imaginary terms in the solution confirms the well known fact that no energy gets dissipated by radiation of waves in this case.

2. For exciting frequencies higher than the fundamental natural frequency of the layer, the power of the exponential functions is purely imaginary for the first term of the series, and either real and negative or purely imaginary for the higher order terms. Accordingly, superimposed on the monotonically varying function of $\xi$ in this case, there are harmonic functions of non-decaying constant amplitudes that persist even as $\xi \rightarrow \infty$. In particular, the far-field motion consists of the superposition of the shear-beam motion and the motion due to waves radiated from the wall.

3. For exciting frequencies equal to any of the natural frequencies of the layer (i.e., $\phi_{n}=1$ or $\left.\phi_{1}=1,3,5, \ldots\right)$, the response of the system becomes infinite. Soil material damping naturally limits these responses to finite values.

2.2.1 Vertical Displacements. On substituting Eq. 31 into Eq. 8, integrating with respect to $\eta$ and satisfying Eq. 15, the vertical displacement $v$ is found to be given by

$$
v(\xi, \eta, t)=v(\xi, \eta) e^{i \omega t}
$$

in which

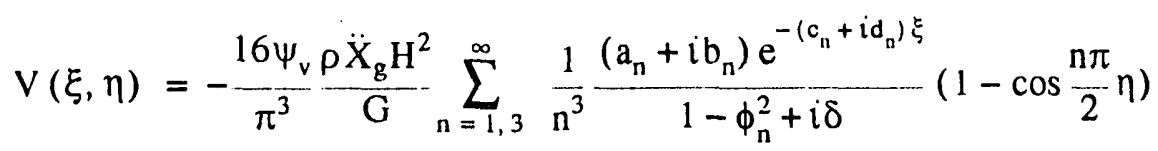

and

$$
\Psi_{v}=\frac{v}{\sqrt{(1-v)(2-v)}}
$$

2.2.2 Normal and Shearing Stresses. These quantities may similarly be expressed as

$$
\sigma_{x}(\xi, \eta, t)=\sigma(\xi, \eta) e^{i \omega t}
$$

and

$$
\tau_{x y}(\xi, \eta, t)=\tau(\xi, \eta) e^{i(\omega t}
$$

and on substituting Eq. 31 into Eq. 9, and Eqs. 31 and 35 into Eq. 4, the following expressions are obtained for the complex-valued stress amplitudes $\sigma(\xi, \eta)$ and $\tau(\xi, \eta)$ : 


$$
\sigma(\xi, \eta)=-\frac{8 \Psi_{\sigma}}{\pi^{2}} \rho \ddot{X}_{g} H(1+i \delta) \sum_{n=1,3}^{\infty} \frac{1}{n^{2}} \frac{\left(a_{n}+i b_{n}\right) e^{-\left(c_{n}+i d_{n}\right) \xi}}{1-\phi_{n}^{2}+i \delta} \sin \frac{n \pi}{2} \eta
$$

in which

$$
\psi_{\sigma}=\frac{\Psi_{\mathrm{o}}^{2}}{\Psi_{\mathrm{e}}}=\frac{2}{\sqrt{(1-v)(2-v)}}
$$

and

$$
\begin{array}{r}
\tau(\xi, \eta)=-\frac{8}{\pi^{2}} \rho \ddot{X}_{g} H \sum_{n=1,3}^{\infty} \frac{1}{n^{2}}\left[\frac{(1+i \delta)\left(1-e^{-\left(c_{n}+i d_{n}\right) \xi}\right)}{1-\phi_{n}^{2}+i \delta} \cos \frac{n \pi}{2} \eta\right. \\
\left.-\frac{v}{2-v} e^{-\left(c_{n}+i d_{n}\right) \xi}\left(1-\cos \frac{n \pi}{2} \eta\right)\right]
\end{array}
$$

For $\xi=0$, i.e., for points on the wall-medium interface, the first group of terms within the summation of Eq. 41 vanish, the exponential terms in the second group of terms become unity, and the shearing stresses $\tau$, like the corresponding displacements defined by Eq. 35, turn out to be generally nonzero and proportional to Poisson's ratio, $v$. It follows that the presumed in-plane interface condition is, as already noted, neither smooth nor fully bonded but intermediate in nature.

2.2.3 Wall Pressures and Forces. The amplitudes of the dynamic wall pressures, $\sigma_{w}(\eta)$, are obtained from Eq. 39 simply by deleting the exponential term. With the pressure amplitudes established, the amplitudes of the total wall force or base shear per unit of length of the wall, $Q_{b}$, and of the corresponding bending moment, $\mathrm{M}_{\mathrm{b}}$, are determined by integration to be

$$
\begin{aligned}
\mathrm{Q}_{b}= & \int_{0}^{1} \sigma_{w}(\eta) H d \eta \\
& =-\frac{16 \Psi_{\sigma}}{\pi^{3}} \rho \ddot{X}_{g} H^{2}(1+i \delta) \sum_{n=1,3}^{\infty} \frac{1}{n^{3}} \frac{a_{n}+i b_{n}}{1-\phi_{n}^{2}+i \delta}
\end{aligned}
$$

and

$$
\begin{aligned}
M_{b}=\int_{0}^{1} \sigma_{w}(\eta) H^{2} \eta d \eta \\
\quad=-\frac{32 \Psi_{\sigma}}{\pi^{4}} \rho \ddot{X}_{g} H^{3}(1+i \delta) \sum_{n=1,3}^{\infty} \frac{(-1)^{\frac{n-1}{2}}}{n^{4}} \frac{a_{n}+i b_{n}}{1-\phi_{n}^{2}+i \delta}
\end{aligned}
$$

in which the negative signs indicate that these forces are induced by compressive wall pressures. 
For an undamped medium with $\delta=0$ for which $a_{n}+i b_{n}=\sqrt{1-\phi_{n}^{2}}$, the wall pressure amplitudes. $\sigma_{w}(\eta)$, and Eqs. 42 and 43 reduce to

$$
\begin{aligned}
& \sigma_{w}(\eta)=-\frac{8 \Psi_{\sigma}}{\pi^{2}} \rho \ddot{X}_{g} H^{2} \sum_{n=1,3}^{\infty} \frac{1}{n^{3}} \frac{1}{\sqrt{1-\phi_{n}^{2}}} \sin \frac{n \pi}{2} \eta \\
& Q_{b}=-\frac{16 \psi_{\sigma}}{\pi^{3}} \rho \ddot{X}_{g} H^{2} \sum_{n=1,3}^{\infty} \frac{1}{n^{3}} \frac{1}{\sqrt{1-\phi_{n}^{2}}}
\end{aligned}
$$

and

$$
M_{b}=-\frac{32 \psi_{\sigma}}{\pi^{4}} \rho \ddot{X}_{g} H^{3} \sum_{n=1,3}^{\infty} \frac{(-1)^{\frac{n-1}{2}}}{n^{4}} \frac{1}{\sqrt{1-\phi_{n}^{2}}}
$$

Note that for $\phi_{n}>1$ (values of $\omega>n \phi_{1}$ ), the terms involving the square-root sign in these equations become imaginary. Note further that the terms within the summation signs in Eqs. 42 through 46 are independent of Poisson's ratio. Accordingly, the dependence of the wall pressures and associated forces on $v$ is reflected fully in the factor $\psi_{\sigma}$ defined by Eq. 40 . Incidentally, for the solution based on the assumption of vanishing vertical displacements, this factor must be replaced by the factor $\chi_{0}$ defined by Eq. 16.

\subsection{Transient Response}

With the harmonic response of the system established, the response to an arbitrary transient excitation is evaluated by the Discrete Fourier Transform (DFT) method in combination with the Fast Fourier Transform (FFT) algorithm [24]. In the application of this procedure, the duration of the forcing function should be increased by the addition of a sufficiently long band of zeros [25] to eliminate the aliasing errors that may be introduced. For the solutions presented herein, the duration of this band was taken equal to either the duration of the forcing function or 10 times the fundamental natural period of the layer idealized as a cantilever shear-beam. 


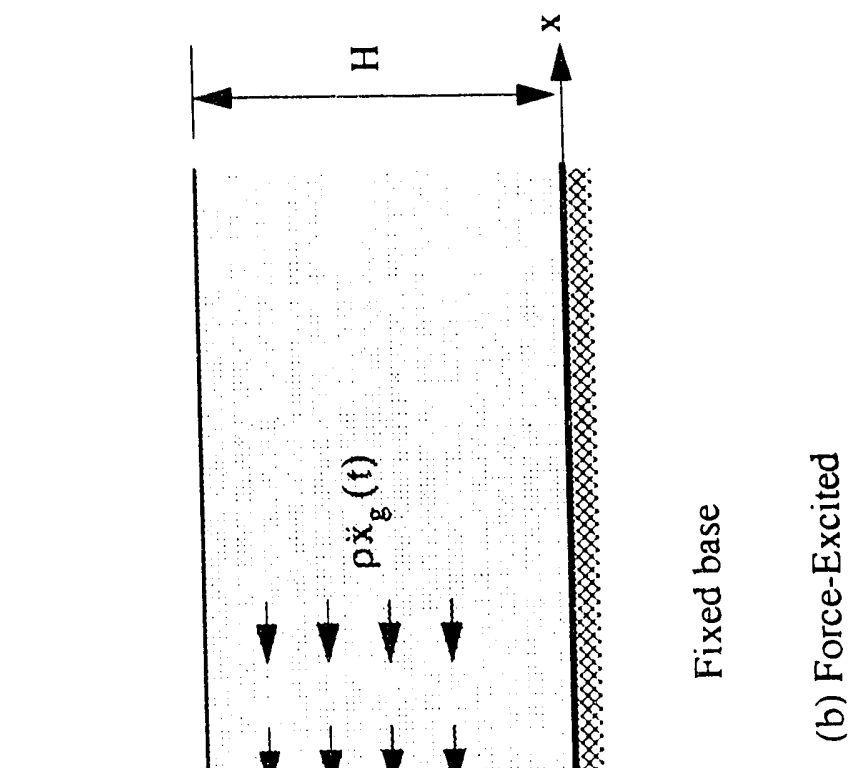

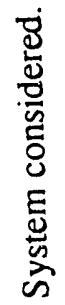

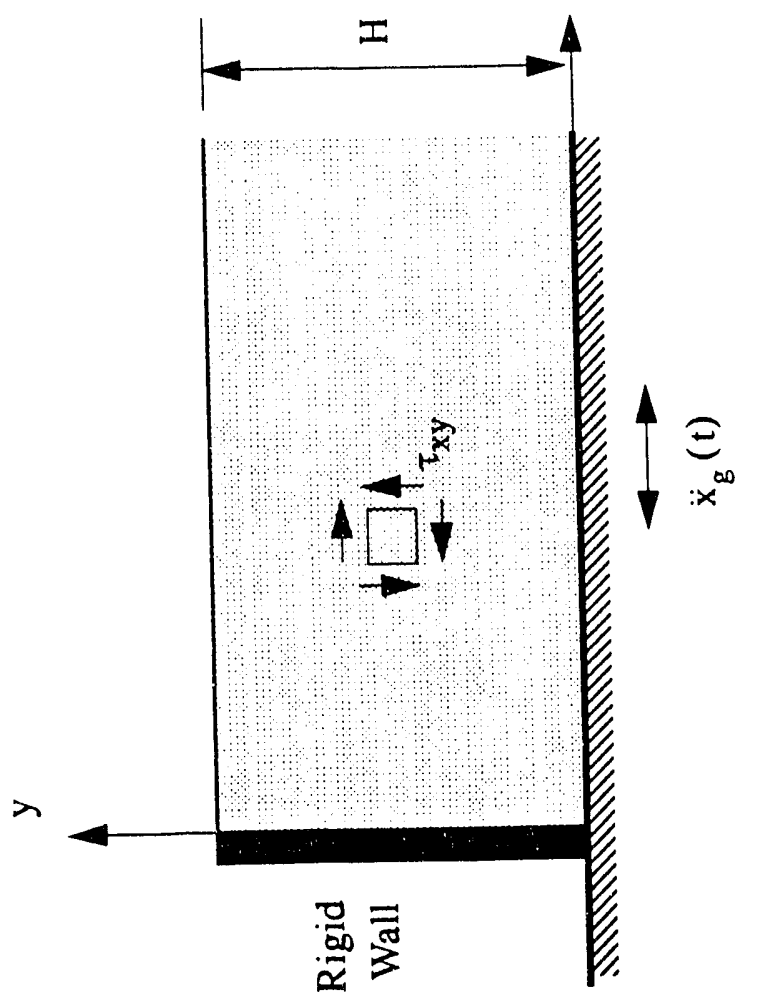

ב

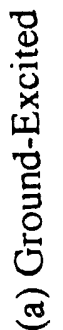




\section{SECTION 3}

\section{PRESENTATION AND ANALYSIS OF RESULTS}

\subsection{Static Effects}

It is desirable to begin by examining the responses obtained for harmonic excitations the frequencies of which are small compared to the fundamental natural frequency of the layer (i.e., for values of $\phi_{1} \rightarrow 0$ ). Such excitations and the resulting effects will be referred to as static, a term which should not be confused with that normally used to represent the effects of gravity forces. In the equivalent, force-excited version of the problem shown in Fig. 2.1(b), the static excitation is represented by a set of horizontal body forces of constant intensity $-\rho \ddot{X}_{\mathrm{g}}$.

The maximum static wall pressure. $\left(\sigma_{w}^{\text {st }}\right)_{\max }$, is attained at the top of the wall, and it is determined from Eq. 44 to be

$$
\left(\sigma_{w}^{s t}\right)_{\max }=-0.742 \Psi_{\sigma} \rho \ddot{X}_{g} H
$$

The maximum shear and bending moment in the wall occur at the base, and their values per unit of length, $Q_{b}^{s t}$ and $M_{b}^{s t}$, are determined from Eqs. 45 and 46 to be

$$
Q_{b}^{s t}=-0.543 \psi_{\sigma} \rho \ddot{X}_{g} H^{2}
$$

and

$$
M_{b}^{s t}=-0.325 \psi_{\sigma} \rho \ddot{X}_{g} H^{3}
$$

For Poisson's ratio $v=0.3,\left(\sigma_{w}^{s t}\right)_{\max }=-1.361 \rho \ddot{\mathrm{X}}_{\mathrm{g}} \mathrm{H}$, which equals the value of the total body force exerted over a soil strip of unit thickness and length $1.361 \mathrm{H}$. Similarly, the corresponding base shear of $0.995 \mathrm{p} \ddot{\mathrm{X}}_{\mathrm{g}} \mathrm{H}^{2}$ is approximately equal to the body force exerted over a square of side length equal to the wall height. These results suggest that the wall pressures and forces in this case are controlled by the action of the medium in the immediate vicinity of the wall rather than by its far-field action.

As a measure of the accuracy of the solutions that have been presented, the base shears for the wall computed from Eq. 48 for different values of Poisson's ratio for the medium are compared in Fig. 3.1 with those obtained from Wood's more rigorous solution [11]. The latter solution is strictly valid for a 
layer of finite length. L. retained by a wall with a smooth interface at each end. For the numerical data reported here, $\mathrm{L}$ was taken as $10 \mathrm{H}$, a value which is believed to adequately approximate the behavior of the semi-infinite layer, and approximately 70 terms were used in the series expressing the variation of effects in the long direction. Also included in Fig. 3.1 are the base shears computed on the assumption of no vertical displacements for the layer. The latter values are obtained from Eq. 48 merely by replacing $\Psi_{\sigma}$ by $\chi_{0}$. The following interrelationships are observed:

1. Over the full range of $v$ values, the results based on the $\sigma_{y}=0$ assumption are in very good agreement with the exact results.

2. For values of $v \leq 1 / 3$, the same also is true of the results based on the $v=0$ assumption. However, as $v \rightarrow 0.5$, the accuracy of the latter solution deteriorates rapidly and ceases to be acceptable.

Mention has already been made of the fact that the solution presented herein does not satisfy the condition of zero shearing stress at the free boundary. Study of Eq. 41 reveals that the unsatisfied shearing stress attains its maximum value at the junction of the surface and the wall, and that it decays rapidly with increasing distance from the wall. Furthermore, its maximum value turns out to be independent of the exciting frequency and generally small compared to the normal pressure exerted on the wall. For the statically excited system, the ratio of the amplitudes of the two stresses, determined approximately from Eqs. 39 and 41 by considering only the first terms of the series, is

$$
\frac{\tau(0,1)}{\sigma(0,1)}=\frac{v}{2} \sqrt{1-v}
$$

The maximum value of this ratio occurs for $v=0.5$ and equals 0.144 ; the value for $v=0.3$ is only 0.096 . It is concluded that the violation of this boundary condition has only a minor influence on the magnitudes of the wall pressures and associated forces, the quantities of primary concern in this study.

Using the finite elements method of analysis, Wood has also evaluated the static wall pressures considering complete bonding between the wall and the soil. [p. 60 of Ref. 11]. The results were found to be in good agreement with those obtained for the smooth interface condition, except for over a very narrow region near the surface, for which the solution for the rough interface exhibits a singularity which cannot, of course, develop in a real soil. This finding provides further support for the reliability of the solutions presented herein for which the in-plane condition at the wall-medium interface is neither smooth nor fully bonded.

\subsection{Results For Harmonic Response}

3.2.1 Magnitude and Distribution of Wall Pressures. The complex-valued amplitude of the dynamic wall pressure, $\sigma_{w}(\eta)$, may conveniently be expressed in the form 


$$
\sigma_{w}(\eta)=-\left(g_{1}+i g_{2}\right) \psi_{\sigma} \rho \ddot{X}_{g} H
$$

in which $g_{1}$ and $g_{2}$ are dimensionless factors that depend on the position coordinate $\eta$, the frequency ratio $\phi_{1}=\omega / \omega_{1}$. and the associated damping factor. $\delta$. The effect of Poisson's ratio is reflected fully in the factor $\psi_{\sigma}$. The real part of this equation represents the restraining effect of the elastic, springlike action of the medium, whereas the imaginary part represents the effect of a damping mechanism analogous to that of a viscous damper. The real-valued amplitude of the pressure is, of course, given by the square root of the sum of squares of the component parts.

The values of $g_{1}$ and $g_{2}$ for the top of the wall are plotted in part (a) $0_{1}^{c}$ Fig. 3.2 as a function of the frequency ratio, $\omega / \omega_{1}$, and the corresponding values of $\sqrt{g_{1}^{2}+g_{2}^{2}}$ are plotted in part (b) of the figure. The material damping factor in these solutions is taken as $\delta=0.1$. The following trends are worth noting in these plots:

1. For $\omega=0$, the factor $g_{2}=0$ and the wall pressure reduces, as it should, to the value defined by Eq. 47.

2. For values of $\omega<\omega_{1}$, the pressure is dominated by the real part of Eq. 51 , whereas for $\omega>\omega_{1}$, it is dominated by the imaginary part. Note in particular, the very rapid and large increase in $g_{2}$ at values of $\omega$ close to $\omega_{1}$. The relative unimportance of the imaginary part of Eq. 51 for $\omega<\omega_{1}$ is consistent with the earlier statement to the effect that, within this range of frequencies, there is no energy dissipation by radiation of waves.

3. The absolute maximum pressure amplitude is attained at $\omega=\omega_{1}$ and it is associated with an amplification factor of 3.39. This is a relatively low value, indicating large overall damping for the system.

The heightwise variation of the factor $\sqrt{g_{1}^{2}+g_{2}^{2}}$ in the expression for the amplitude of the wall pressure is shown in Fig. 3.3(a) for selected values of the frequency ratio, $\omega / \omega_{1}$. It is observed that, for values of $\omega / \omega_{1}$ of the order of 2 or less, the stress amplitudes increase monotonically from zero at the base to a maximum at the top. and that they are dominated by the fundamental mode of vibration of the layer. By contrast, the wavy variations for the larger values of $\omega / \omega_{1}$ reflect the increased contributions of the higher modes of vibration.

The pressure distribution for $\omega=3 \omega_{1}$ (i.e., at the second natural frequency of the layer), may better be appreciated from the plots in Fig. 3.3(b), which identify the real and imaginary parts of the pressure amplitude in addition to the real-valued amplitude. Note that the horizontal scale in this figure is twice as large as that employed in Fig. 3.3(a).

3.2.2 Horizontal Variation of Effects. For the frequency ratios considered previously in Fig. 3.3(a). Fig. 3.4 shows the horizontal variation of the real-valued amplitudes of the horizonta' normal stress at 
the top of the layer, $\left|\sigma_{x}(\xi, 1)\right|$. Similarly, Fig. 3.5 shows the corresponding variations of the real-valued amplitudes of the top displacement relative to the moving base, $! U(\xi, 1) \mid$, and of the shear at the soil-rock interface, $|\tau(\xi, 0)|$. Stresses in these plots are normalized with respect to $\rho \ddot{X}_{\mathrm{g}} \mathrm{H}$, and displacements are normalized with respect to $\rho \ddot{X}_{\mathrm{g}} \mathrm{H}^{2} / \mathrm{G}$. The de data are for a medium with $v=0.3$ and $\delta=0.1$. Unless otherwise indicated, the same medium properties will be considered for all other solutions that follow.

As would be expected, the amplitudes of the normal stress decrease from their maximum or near maximum values at the wall to zero at the far field, whereas those of the shearing stress and displacement increase from zero at the wall to the values associated with the shear-beam action of the layer at the far field. The variations are monotonic for $\omega / \omega_{1}<1$, confirming the absence of radiational effects, and oscillatory for the higher values of $\omega / \omega_{1}$ for which the radiational effects are important. 'The rate of decay of the normal stress with increasing distance from the wall is quite rapid for the small values of $\omega / \omega_{1}$ and only moderate for the higher values. This suggests that whereas the wall pressures at low frequencies are, as already indicated. controlled by the action of the medium in the immediate vicinity of the wall, those at high frequencies are also influenced by the far-fielo action of the medium.

3.2.3 Base Shear and Moment. The real-valued amplitude of the base shear per unit length of the wall, $\left|Q_{b}\right|$, is plotted in Fig. 3.6 as a function of the frequency ratio $\omega / \omega_{1}$ for three different values of the material damping factor for the medium. $\delta$. Poisson's ratio for the medium in these solutions is again taken as $v=0.3$. The results are normalized with respect to $\rho \ddot{X}_{\mathrm{g}} \mathrm{H}^{2}$.

As would be expected, all curves start at the left from the static value of $0.995 \rho \ddot{X}_{g} H^{2}$ identified previously, and the curve for the undamped system becomes infinite at exciting frequencies equal to the natural frequencies of the layer. Soil material damping reduces significantly the resonant peaks, particularly those corresponding to the higher modes of vibration. For $\delta=0.1$, the amplification factor for the first resonant peak is only 3.05 , whereas the second resonant peak is hardly noticeable.

The very significant effect of damping on the higher resonant peaks is associated with the fact that, except for the totally undamped system, the fundamental mode of vibration is the dominant contributor to the response even for high-frequency excitations. This is demonstrated in Fig. 3.7, in which the exact amplitudes of the base shear for a wall retaining a medium with $\delta=0.1$ are compared with those obtained considering the contribution of the fundamental mode of vibration only. The excellent agreement between the two sets of results indicates that the one-mode approximation should be satisfactory for transient excitations as well.

The amplitude of the base moment per unit length of the wall may conveniently be expressed as the product of the base-shear amplitude and an appropriate height, $h$. If the response were contributed solely by the fundamental mode of vibration, considering that this mode varies as a half-sine from 
zero at the base to a maximum value at the free surface, the height $h$ would have been $(2 / \pi) \mathrm{H}=0.637 \mathrm{H}$. The exact values, identified in Fig. 3.8, are not materially different, except for a totally undamped system at exciting frequencies close to the second natural frequency of the layer.

\subsection{Solutions for Transient Response}

The response of the medium was also evaluated for the first $6.3 \mathrm{sec}$ of the $\mathrm{N}-\mathrm{S}$ component of the ground motion recorded during the $1940 \mathrm{El} \mathrm{Centro,} \mathrm{California} \mathrm{earthquake.} \mathrm{The} \mathrm{acceleration,} \mathrm{velocity}$ and displacement traces of this record have been presented before in [26]. The peak value of the ground acceleration is $\ddot{x}_{\mathrm{g}}=0.312 \mathrm{~g}$, and the corresponding values of the velocity and displacement are $\dot{x}_{g}=14.02 \mathrm{in} / \mathrm{sec}$ and $x_{g}=8.29$ in.

The upper solid line in Fig. 3.9 defines the absolute maximum value of the base shear per unit length of the wall induced by the El Centro ground motion for a medium characterized by $v=0.3$ and $\delta=0.10$. The results are plotted as a function of the fundamental cyclic natural frequency of the medium, defined by

$$
f_{1}=\frac{v_{s}}{4 H}
$$

and they are normalized with respect to $\rho \ddot{x}_{g} H^{2}$. It should be noted that the right part of this figure, which defines high-frequency, stiff systems, corresponds to the left parts of Figs. 3.2, 3.6 and 3.7, and that the left part of Fig. 3.9 corresponds to the right parts of the previous figures.

As a measure of the values of $f_{1}$ that may be encountered in practice, it is noted that for values of $v_{s}$ in the range between 400 and $1600 \mathrm{ft} / \mathrm{sec}$ and values of $\mathrm{H}$ in the range between 10 and $50 \mathrm{ft}$, the value of $f_{1}$ falls in the range of $2 \mathrm{cps}$ to $40 \mathrm{cps}$.

The responses of high-frequency systems, with values of $f_{1}$ of the order of $30 \mathrm{cps}$ or more, is effectively static, and the maximum base shear per unit of length of the wall in this case is approximately $\rho \ddot{x}_{g} \mathrm{H}^{2}$. With decreasing system frequency, the peak response increases but, as would be expected of a highly damped system, the increase is limited to a factor of less than 1.5. For highly flexible, very low-frequency systems, the maximum effects are naturally less than those obtained under static conditions of excitation.

The upper dashed line in Fig. 3.9 defines the values of the maximum base shear computed considering the contribution of only the fundamental mode of vibration of the medium. As anticipated from the responses of the harmonically excited systems examined earlier, the agreement with the more nearly exact solution is indeed excellent. If follows that the maximum values of the wall pressures may, for all practical purposes, be considered to increase as half-sine waves from zero at the base to a maximum value at the top, and the maximum base moment may be computed as the product of the base 
shear and the height $h=0.637 \mathrm{H}$. The significance of the lower two curves in Fig. 3.9 is identified in the following section.

Before proceeding to the next section, it is desirable to reiterate that, when appropriately adjusted, the data for the wall pressures and associated forces presented in Figs. 3.2, 3.3 and 3.6 through 3.9 are also valid for the solution based on the assumption of vanishing vertical displacements. It is only necessary to replace the factor $\Psi_{\sigma}$ in Eqs. 42 through 49 and Eq. 51 by the factor $\chi_{o}$ defined by Eq. 16. 


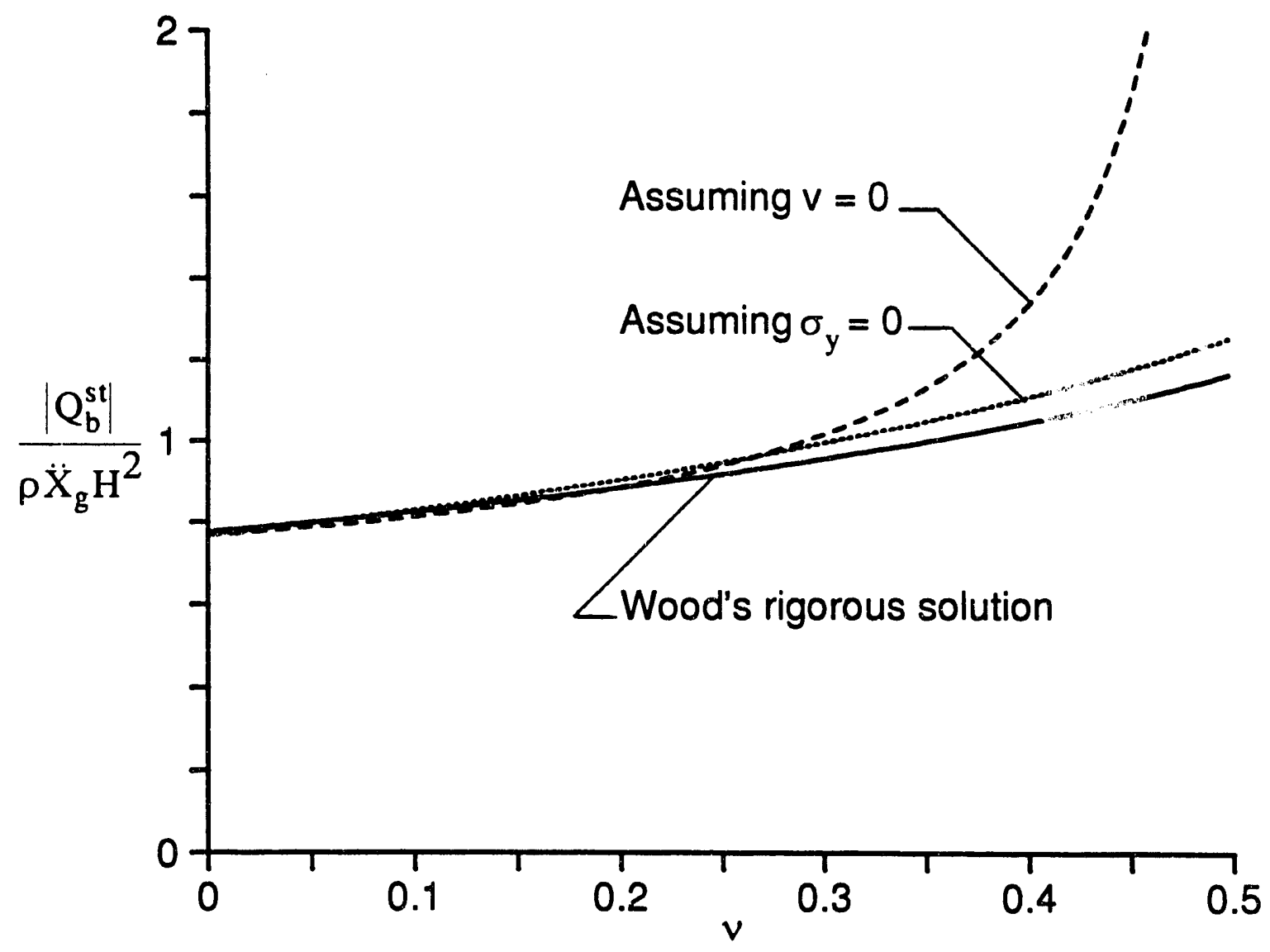

Figure 3.1 Comparison of static values of base shear in wall computed by different procedures. 


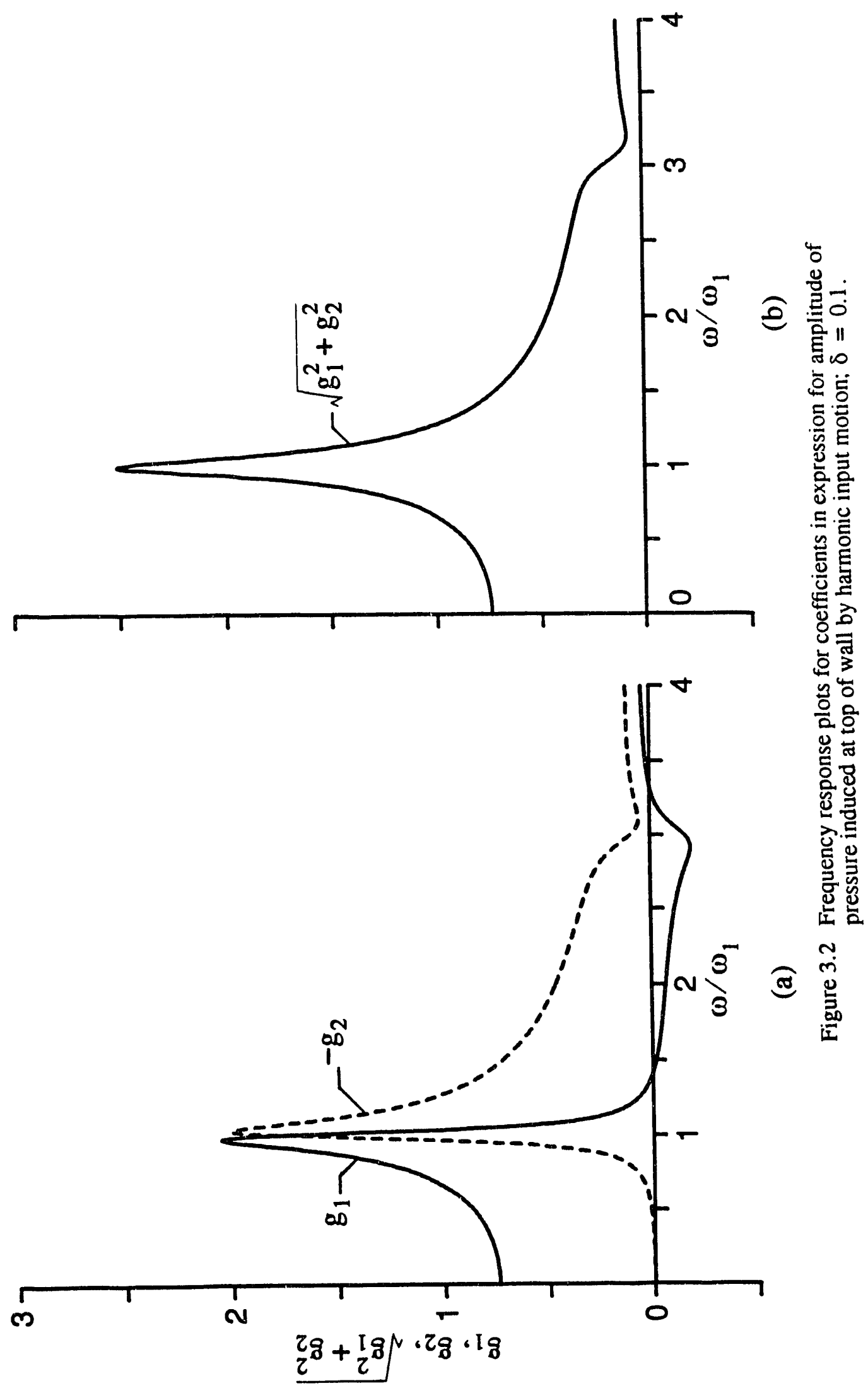




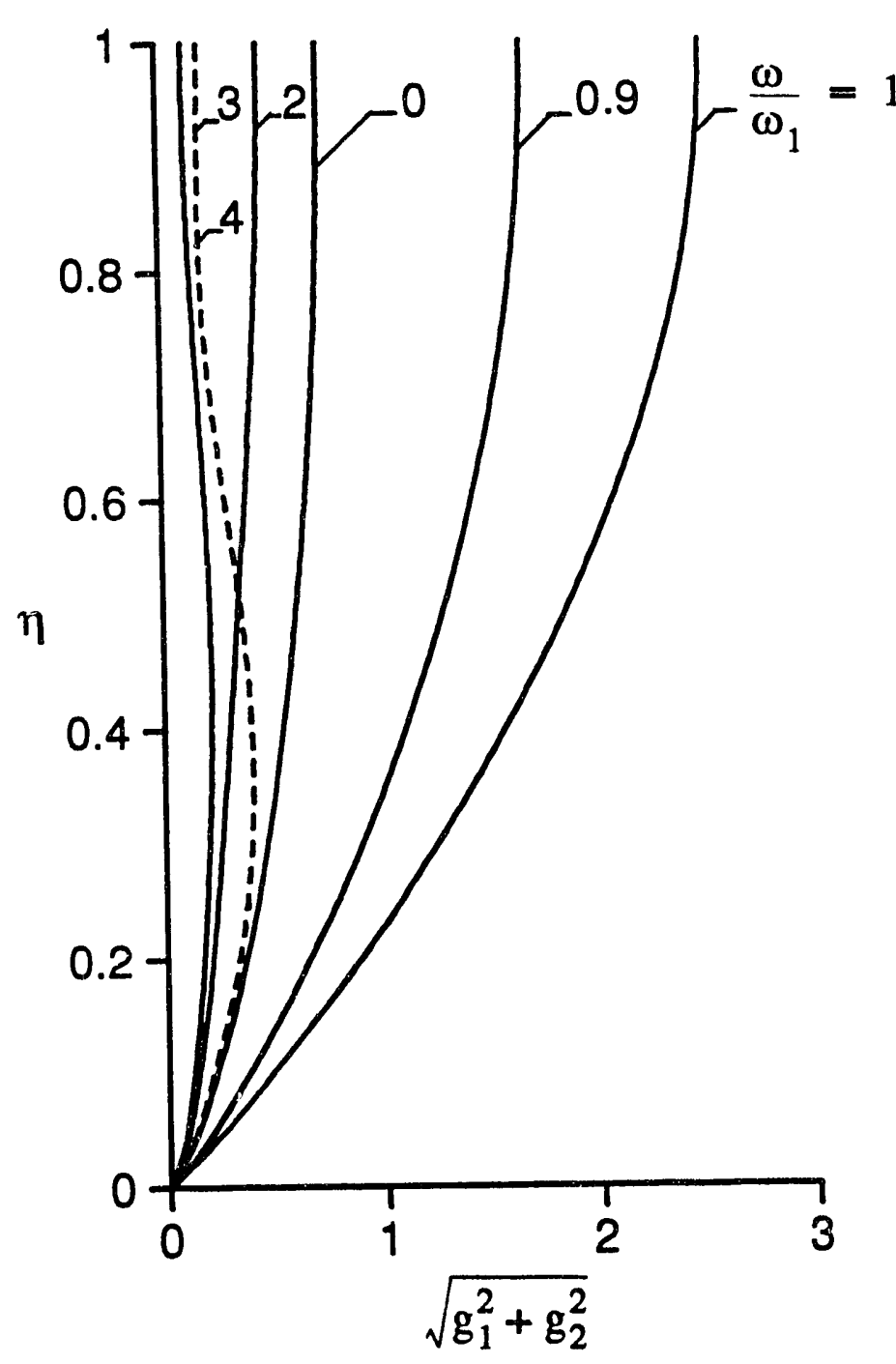

(a)

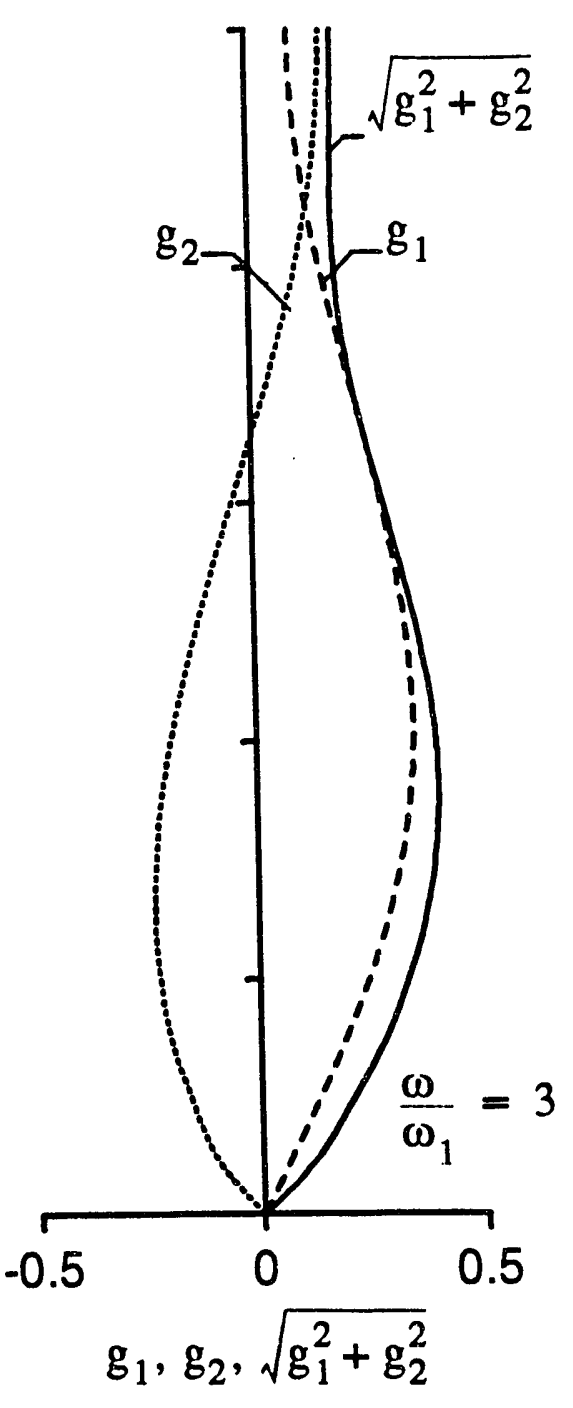

(b)

Figure 3.3 Heightwise variations of coefficients in expression for wall pressure induced by harmonic input motion; $\delta=0.1$. 


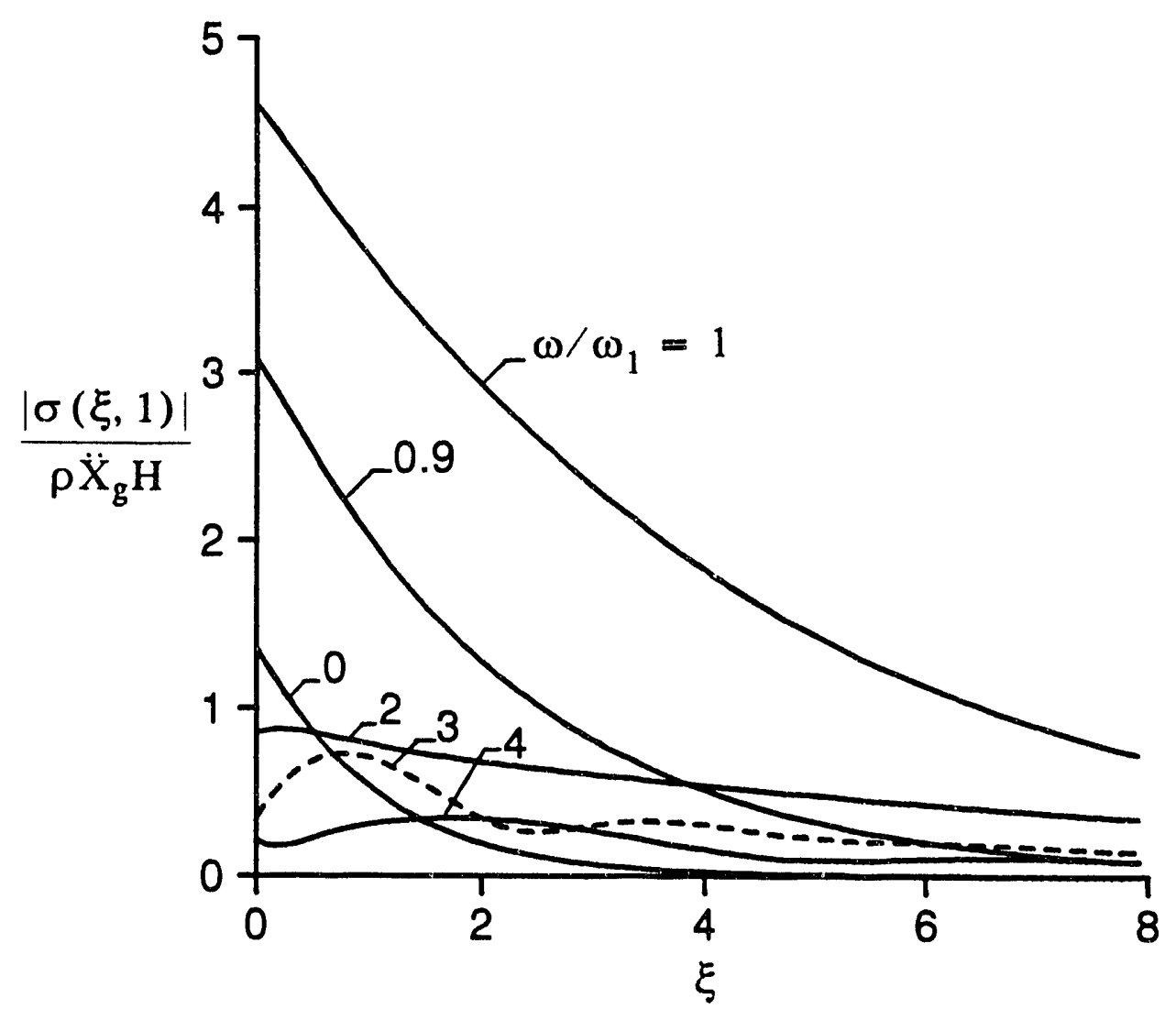

Figure 3.4 Horizontal variations of horizontal normal stresses induced at top of layer by harmonic base motion: $v=0.3, \delta=0.1$. 

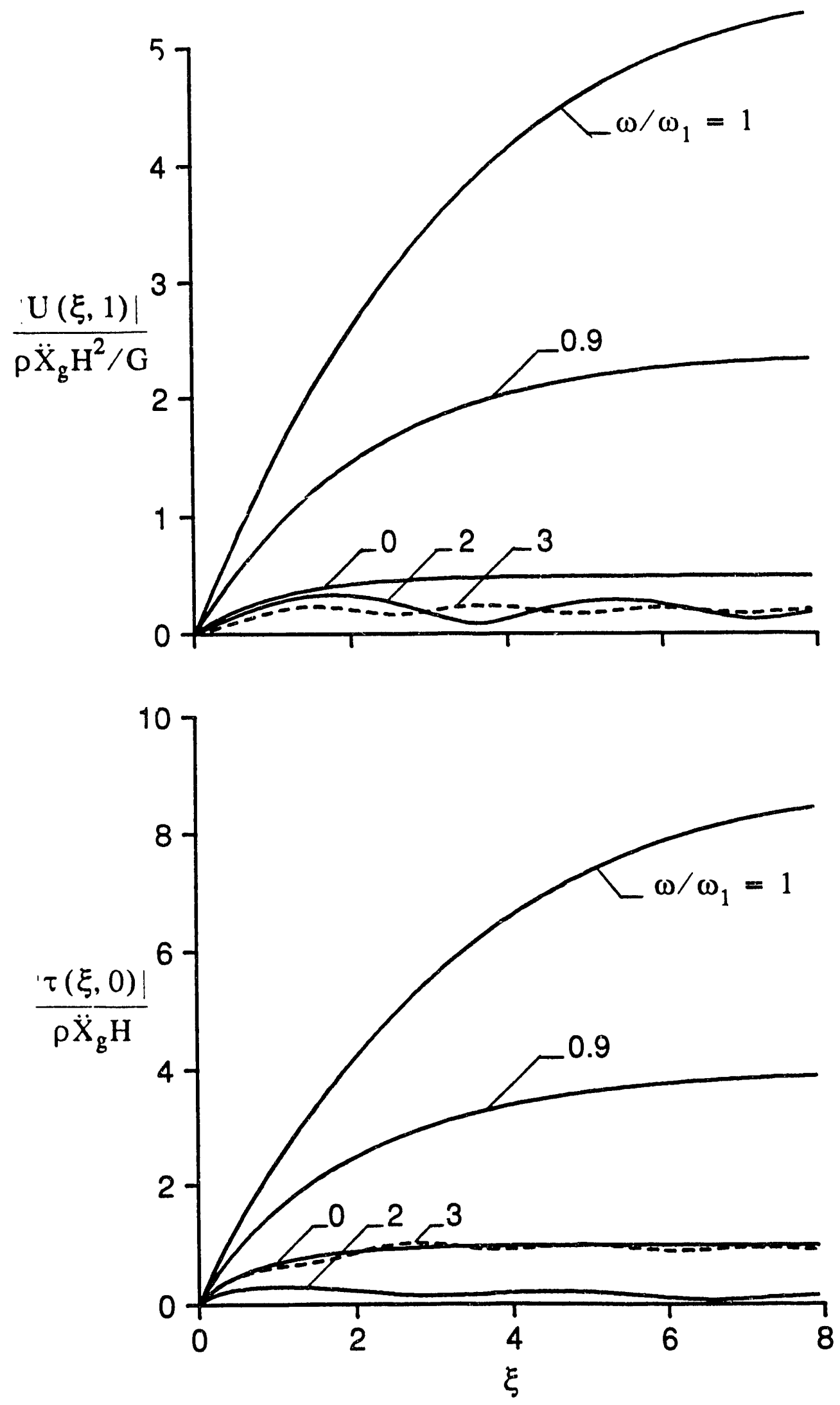

Figure 3.5 Horizontal variations of top displacements and base shearing stresses induced by harmonic base motions; $v=0.3, \delta=0.1$. 


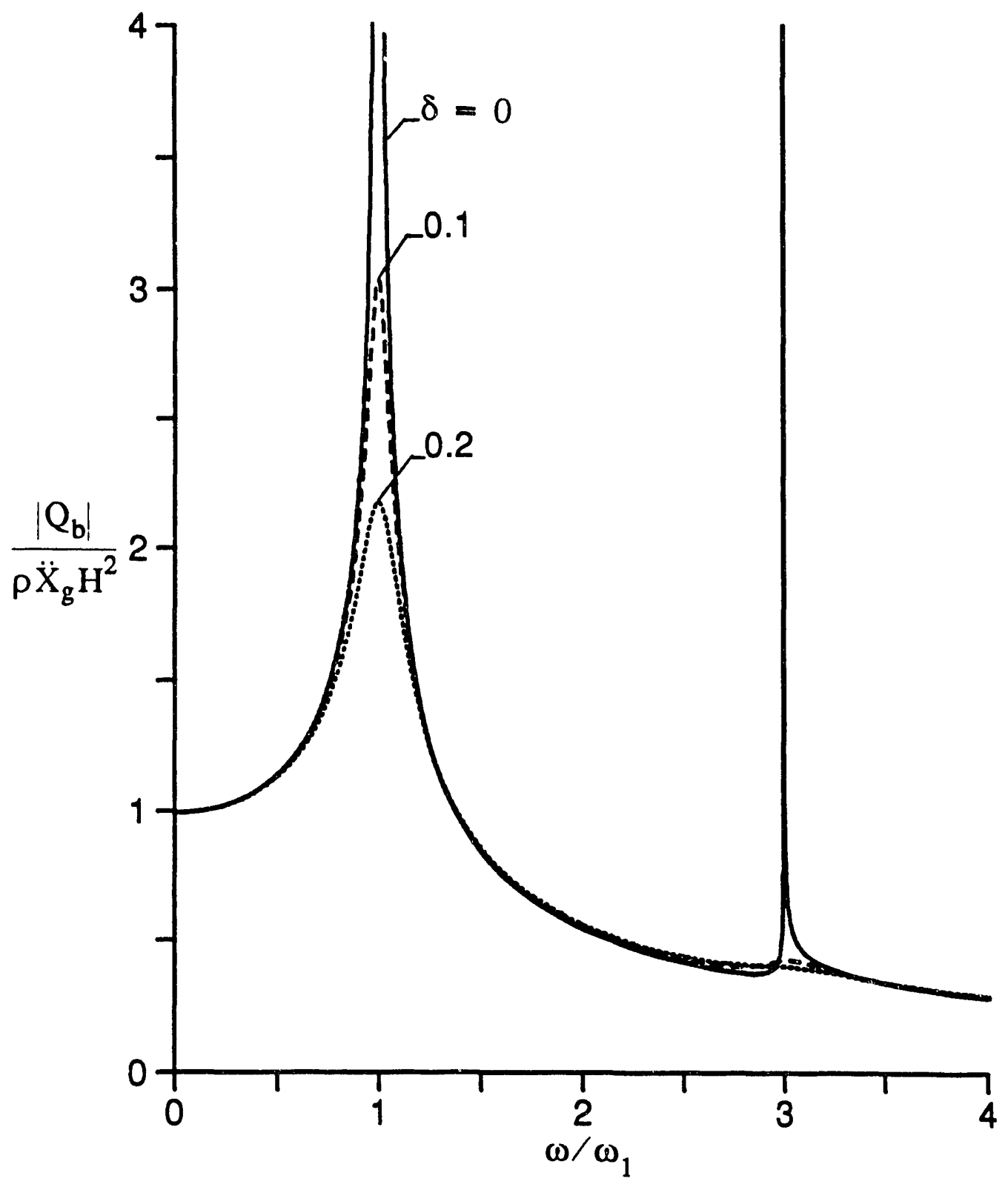

Figure 3.6 Effect of soil material damping on amplitudes of base shear induced in wall of harmonically excited systems: $v=0.3$. 


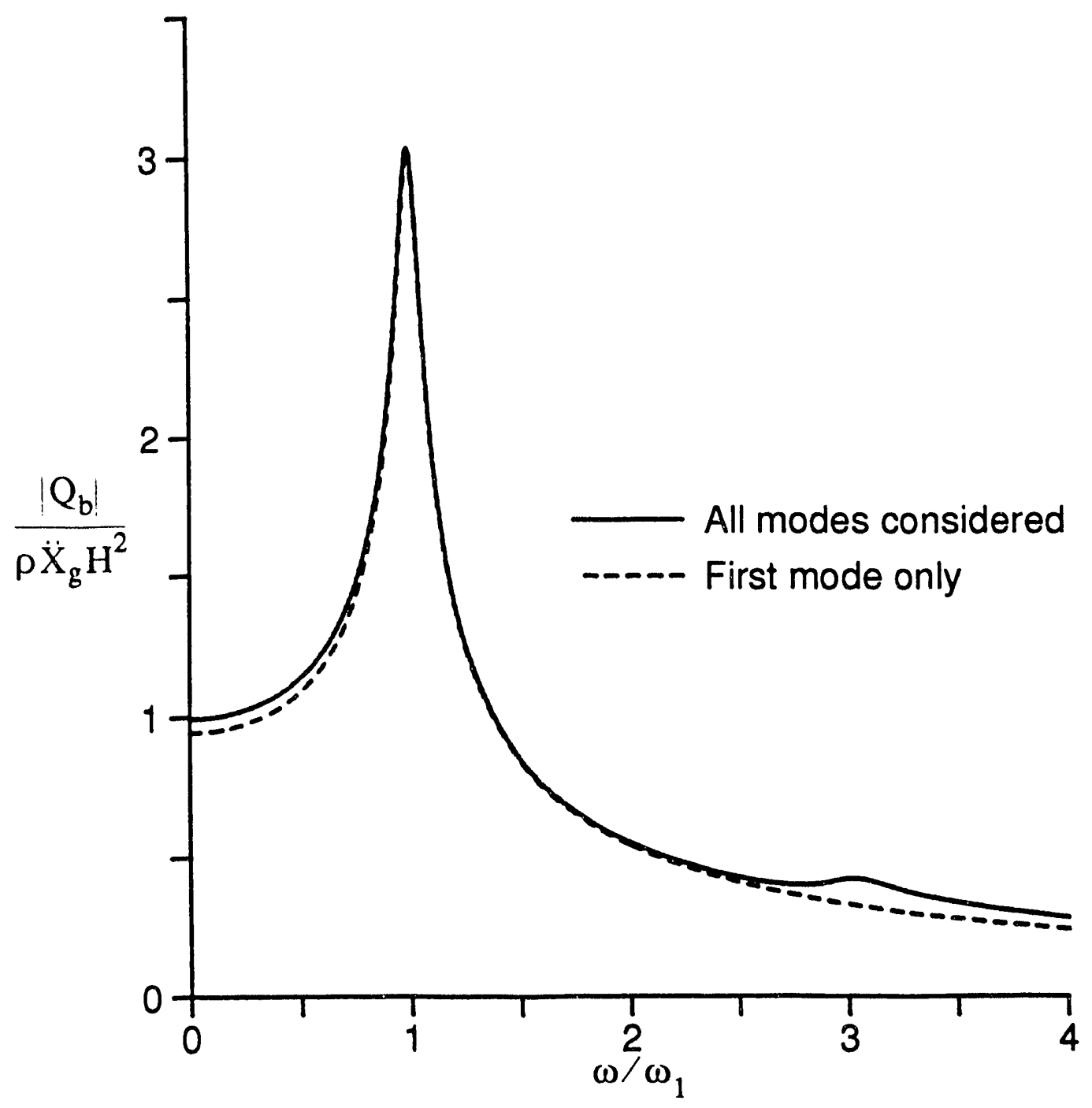

Figure 3.7 Comparison of frequency response curves for base shear in wall computed by considering one and all modes of vibration; $v=0.3, \delta=0.1$. 


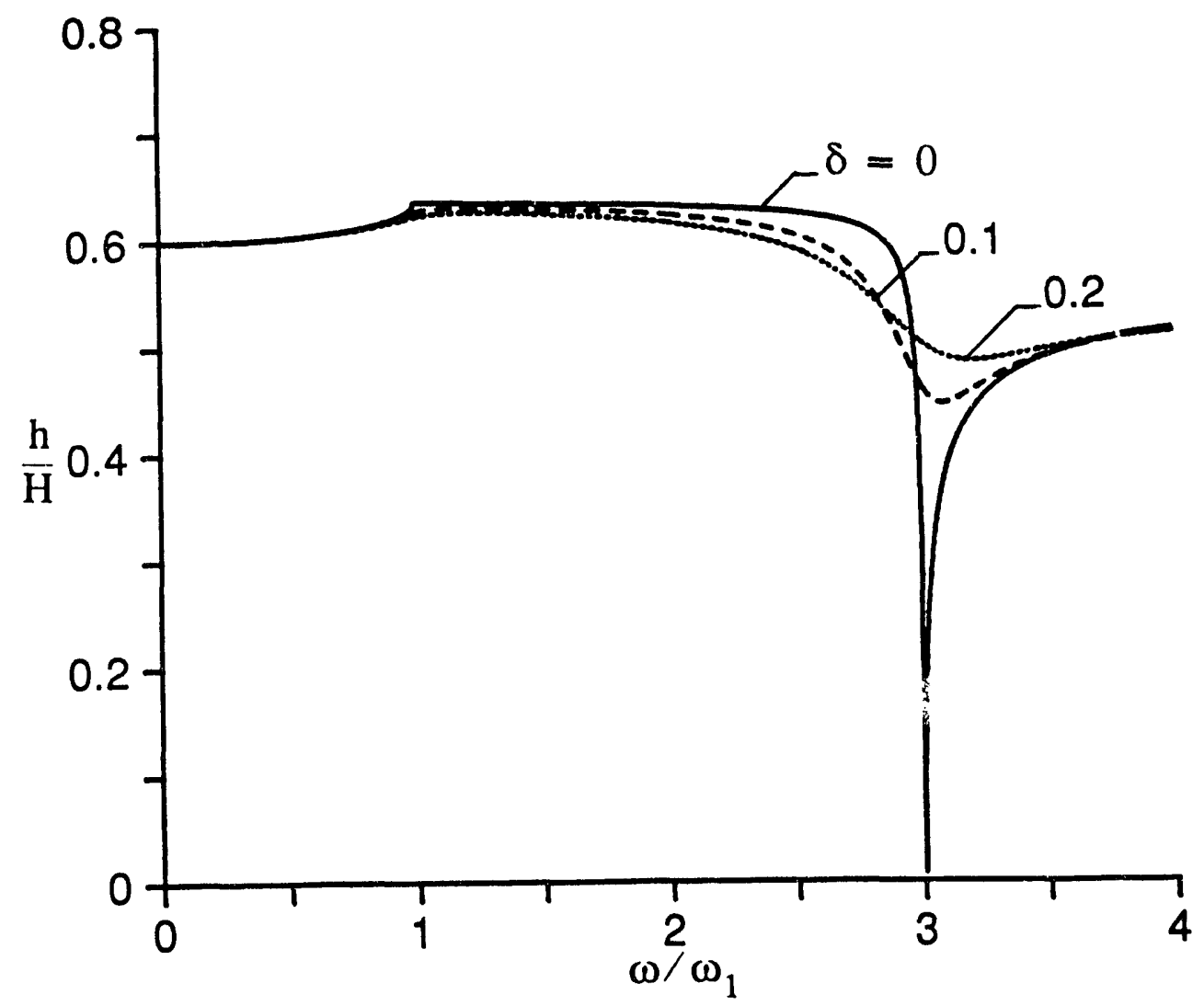

Figure 3.8 Height of resultant of total wall force induced by harmonic base excitation: $v=0.3$. 


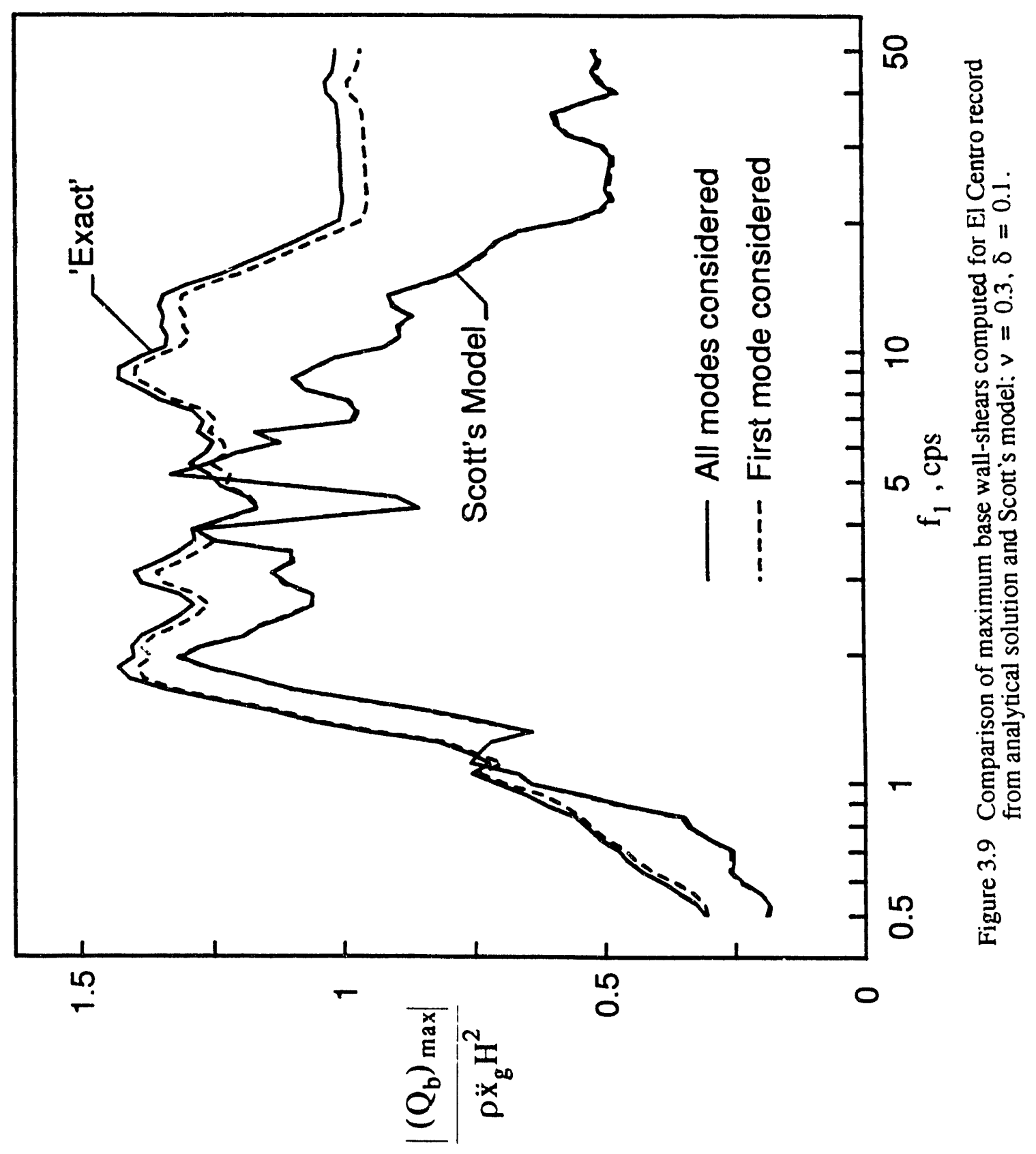




\section{SECTION 4 \\ MODELING OF SYSTEM}

\subsection{Scott's Model}

The accuracy of Scott's model is assessed in this section for the special case of the semi-infinite stratum. The model is shown in Fig. 4.1; it consists of a uniform cantilever shear-beam that represents the far-field action of the layer and a set of distributed linear horizontal springs connecting the shear-beam to the wall. The material properties and height of the beam are taken equal to those of the stratum, and the stiffness of the springs per unit of length and height of the wall, $k_{s}$, is taken as

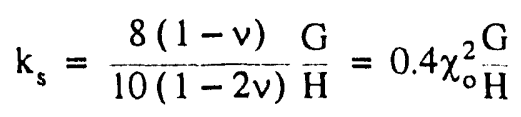

Both the wall and shear-beam are presumed to be excited by the same ground motion. Note that the only damping for the model is that involved in the shear-beam itself.

Considering that the relative displacement amplitudes for a harmonically excited shear-beam are defined by Eq. 32, the amplitudes of the wall pressures for the model are given by the product of this equation and $\mathrm{k}_{\mathrm{s}}$ as

$$
\sigma_{w}(\eta)=-\frac{6.4 \chi_{0}^{2}}{\pi^{3}} \rho \ddot{X}_{g} H \sum_{n=1,3}^{\infty} \frac{1}{n^{3}} \frac{1}{1-\phi_{n}^{2}+i \delta} \sin \frac{n \pi}{2}-\eta
$$

The amplitudes of the base shear and base moment per unit length of the wall are similarly given by

$$
Q_{b}=-\frac{12.8 \chi_{o}^{2}}{\pi^{4}} \rho \ddot{X}_{g} H^{2} \sum_{n=1,3}^{\infty} \frac{1}{n^{4}} \frac{1}{1-\phi_{n}^{2}+i \delta}
$$

and

$$
M_{b}=-\frac{25.6 \chi_{0}^{2}}{\pi^{5}} p \ddot{X}_{g} H^{3} \sum_{n=1,3}^{\infty} \frac{(-1)^{\frac{n-1}{2}}}{n^{5}} \frac{1}{1-\phi_{n}^{2}+i \delta}
$$

There are several shortcomings to this model: 
1. The rationale and accuracy of Eq. 53 are uncertain. Considering that the wall pressures and forces are proportional to $\mathrm{k}_{\mathrm{s}}$, any errors involved in the specification of this stiffness affect directly the resulting responses. It should also be noteu that as $v$ tends to $0.5, \mathrm{k}_{\mathrm{s}}$ and hence the wall pressures and the associated forces become infinite.

2. The ground motion in the model is effectively presumed to be resisted by shearing action of the medium in the far field, and by extensional, column-like action between the far field and the wall. No provision is made for the shearing resistance of the portion of the medium in the immediate vicinity of the wall, which, based on the information presented in previous sections, may affect importantly the resulting wall pressures.

3. The model does not provide for the radiational damping capacity of the medium; as a result, it may significantly overestimate the responses of the system at exciting frequencies close to the natural frequencies of the stratum.

In Fig. 4.2, the real and imaginary parts of the frequency response curves for the amplitude of the pressure at the top of the wall computed from Eq. 47 are presented along with the corresponding plots obtained by use of the Scott model. As before. Poisson's ratio and the material damping factor in these solutions are taken as $v=0.3$ and $\delta=0.1$. Comparison of the two sets of results reveals the following:

1. The static value of the pressure amplitude for the model is significantly lower than that obtained by the more rigorous approach, a result indicating that the spring stiffness defined by Eq. 53 is too low:

2. As anticipated, the fundamental resonant peak of the pressure computed by the model is indeed significantly higher than the exact value.

3. The relative magnitudes of the real and imaginary parts in the two solutions are different over the entire range of the frequency parameter. Note, in particular, that whereas the responses of high-frequency systems in the more rigorous solution are dominated by the imaginary part, in Scott's model, they are dominated by the real part.

The differences between the two solutions may better be appreciated from Fig. 4.3 which compares the real-valued amplitudes of the base shear computed by the two approaches. The agreement is clearly less than satisfactory. It is noteworthy, however, that whereas Scott's model overestimates the response at exciting frequencies close to the fundamental natural frequency of the stratum, it underestimates the response at the remaining frequencies. It is conceivable, therefore, that for earthquake ground motions for which the dominant frequencies of its harmonics fall within a band extending to both sides of the fundamental natural period of the stratum, the differences in the responses computed by the two approaches may not be as large as might be inferied from the differences in the ordinates of the plots in Fig. 4.3. 
That this indeed the case is demonstrated in Fig. 3.9, in which the exact maximum values of the base wall-shear induced by the El Centro record are compared with those computed from Scott's model. Within the middle range of the frequencies considered, the agreement between the two sets of results may not be deemed to be unreasonable. However, this agreement is fortuitous: it stems from compensating errors associated with the underestimation of both the stiffness and damping capacity of the system.

If the stiffness of the springs in Scott's model were increased so as to yield the correct, effectively static response at high natural frequencies, it is clear from Fig. 3.9, that the comparison at the other frequencies would deteriorate dramatically. Obviously, this is not a desirable modification of Scott's model.

As would be expected from information already presented, the fundamental mode approximation of the solution based on Scott's model (represented by the lower dashed line in Fig. 3.9) is in excellent agreement with the corresponding exact solution.

\subsection{Proposed Models}

For the purpose of estimating the dynamic wall pressures and the associated base shear and base moment, the action of the soil stiatum may more reliably be modeled by that of the simple oscillator shown in Fig. 4.4. The mass of this model, $\mathrm{m}^{*}$, is determined so that the total wall force or base shear for static conditions of I esponse equals the exact value defined by Eq. 48 . This requires that

$$
\mathrm{m}^{*}=0.543 \psi_{\sigma} \rho \mathrm{H}^{2}
$$

4.2.1 Model with Constant Parameters. The spring stiffness, $\mathrm{k}^{*}$, in this case is determined so that the undamped natural frequency of the model equals the fundamental natural frequency of the medium idealized as a series of vertical shear-beams. The result is

$$
\mathrm{k}^{*}=\mathrm{m}^{*} \frac{\pi^{2}}{4 \mathrm{H}^{2}} \frac{\mathrm{G}}{\rho}=1.339 \psi_{\mathrm{o}} \mathrm{G}
$$

Finally, the damping coefficient, $c^{*}$, is determined so that. for a harmonic excitation of a frequency equal to the natural frequency of the model, the total wall force for the model and the actual system are equal. This requires that the damping of the system, in percent of critical damping $\zeta^{*}$, be defined by

$$
\zeta^{*}=\frac{1}{\left.2 \cdot \mathrm{TR}_{1}\right|^{2}-1}
$$

in which the transmissibility factor, $\left|\mathrm{TR}_{1}\right|$, represents the ratio of the exact real-valued amplitude of 
the total wall force at $\omega=\omega_{1}$ to the corresponding force induced under static conditions of response.

Implicit in the development of Eq. 59 is the assumption that the coefficient of viscous damping, $\mathrm{c}^{*}$, is constant. If, in the spirit of the constant hysteretic form of damping used in the characterization of the hysteretic properties of the medium, the product $\omega \mathrm{c}^{*}$ rather than $\mathrm{c}^{*}$ is considered to be constant, then the damping factor will be inversely proportional to $\omega$. On denoting the latter factor by $\zeta^{*}(\omega)$, one obtains

$$
\zeta^{*}(\omega)=\frac{\zeta^{*}}{\omega / \omega_{1}}
$$

With the total wall force established, the wall pressures are determined considering their magnitudes to increase as a half-sine from the base to the top, and the base moment is determined assuming the force resultant to act at the centroid of the pressures, i.e., at a distance $h=(2 / \pi) \mathrm{H}$ from the base.

The exact response spectrum for the base shear of harmonically excited systems is compared in Fig. 4.5 with the corresponding spectra obtained by the proposed model for each of the two forms of damping referred to above. It is observed that:

1. The agreement between the two sets of results is not particularly good for exciting frequencies other than zero and the fundamental natural frequency of the stratum.

2. The results are insensitive to the forms of damping considered, even as $\omega / \omega_{1}$ tends to zero and the value of $\zeta^{*}(\omega)$ for the constant hysteretic form of damping tends to infinity.

3. The differences between the rigorous and approximate solutions suggest that the stiffness of the spring in the proposed model is not constant as presumed, and that the frequency-dependence of the damping coefficient corresponds to neither of the two forms of damping considered.

4.2.2 Model with Frequency-Dependent Parameiars. The mass of the model, $\tilde{m}$, in this case is defined, as before, by Eq. 57 , but the spring stiffness, $\tilde{k}(\omega)$, and the damping coefficient, $\tilde{c}(\omega)$, are determined so that the harmonic responses of the actual and replacement systems are identical at all frequencies.

Let TR be the complex-valued transmissibility of the actual system at an arbitrary frequency, and $(T R)_{m}$ be the corresponding quantity for the model. The exact transmissibility is determined from Eq. 42 by normalizing the result by the corresponding static value.

With the mass of the model defined by Eq. 57. the static effects for the two systems are identical, and the equality of the dynamic effects may be satisfied by equating the two 1 ansmissibilities. Recalling that 


$$
(\mathrm{TR})_{m}=\frac{\tilde{\mathrm{K}}(\omega)}{\tilde{\mathrm{K}}(\omega)-\tilde{\mathrm{m}} \omega^{2}}
$$

where $\tilde{K}(\omega)=$ the complex-valued impedance of the model defined by

$$
\tilde{K}(\omega)=\tilde{k}(\omega)+i \omega \tilde{c}(\omega)
$$

replacing $(T R)_{m}$ by $T R$, and solving for $\tilde{K}(\omega)$, one obtains

$$
\tilde{\mathrm{K}}(\omega)=\frac{\mathrm{TR}}{\mathrm{TR}-1} \tilde{\mathrm{m}} \omega^{2}
$$

This quantity may conveniently be expressed either as

$$
\tilde{\mathrm{K}}(\omega)=\tilde{\mathrm{k}}_{\mathrm{st}}\left(\tilde{\alpha}+i 2 \tilde{\zeta} \frac{\omega}{\omega_{1}}\right)
$$

or in the form

$$
\tilde{K}(\omega)=\tilde{k}_{\text {st }}(\tilde{\alpha}+i \tilde{\beta})
$$

in which $\tilde{\mathrm{k}}_{\mathrm{st}}=$ the static value of $\tilde{\mathrm{K}}(\omega)$ corresponding to $\omega / \omega_{1}-; 0 ; \tilde{\alpha}=$ a dimensionless stiffness coefficient which is unity at $\omega=0: \tilde{\zeta}=$ the damping of the mod. in percent of critical damping; and $\tilde{\beta}$ is an alternative damping factor, analogous to ine factor $\delta$ used to characterize the soil material damping of the medium in the original system. The factors $\tilde{\alpha}, \tilde{\zeta}$ and $\tilde{\beta}$ are functions of the frequency ratio $\omega / \omega_{1}$ and of the soil material damping factor $\delta$.

In the following, the parameters of the model are determined approximately using for TR the values obtained considering the contribution of only the fundamental mode of vibration of the layer. For this approximation, the mass of the model is given by

$$
\tilde{m}=\frac{16 \psi_{\sigma}}{\pi^{3}} \rho H^{2}
$$

the static spring stiffness is given by

$$
\tilde{\mathrm{k}}_{\mathrm{st}}=\frac{8 \Psi_{\mathrm{o}}}{\pi} \mathrm{G}
$$

and the values of $\tilde{\alpha}, \tilde{\zeta}$ and $\tilde{\beta}$ are as shown in Fig. 4.6. Note that for a non-dissipative medium with $\delta=0$, the stiffness coefficient decreases from unity at $\omega=0$ to about 0.5 at $\omega=\omega_{1}$, then remains constant. In this case, both $\tilde{\zeta}$ and $\tilde{\beta}$ are zero for $\omega \leq \omega_{1}$, and at the high frequencies, they approach asymptotically the values of $\tilde{\zeta}=0.25$ and $\tilde{\beta}=0.5 \omega / \omega_{1}$. For a medium with non-zero values of $\delta$. as $\omega / \omega_{1} \rightarrow 0 \tilde{\beta}$ tends to $\delta$ and $\tilde{\zeta}$ tends to infinity.

It has been observed [27] that the variations with frequency of the dimensionless impedance factors for the model are similar to those reported by Wolf [28] for a semi-infinite rod with exponentially 
increasing cross sectional area. However, the interrelationship of the two problems is not clear at this time.

Had the values of $\tilde{\alpha}, \tilde{\zeta}$ and $\tilde{\beta}$ been determined from the complete solution, rather than its first mode approximation, they would have been found to be more sensitive to variations in the exciting frequency, particularly at frequencies close to the second natural frequency of the layer. It must be emphasized, however, that this model with frequency-dependent characteristics is not proposed as a practical means of analyzing the actual system. but rather as a means of visualizing and explaining its response. After all, the response of the actual system may be evaluated as readily as that of the model.

4.2.3 Model with Modified Frequency-Independent Parameters. Both for the actual system and the simple replacement oscillator with frequency-dependent properties referred to in the preceding section, the evaluation of the response to transient excitations requires the use of Fourier transform or Laplace transform techniques. By contrast, the analysis of the constant-parameter model described earlier may be implemented much more readily by direct integration procedures. It is desirable, therefore, to explore the possibility of adjusting the constant-parameter model so that it better approximates the response of the actual system.

Instead of determining the damping of the model so that its response at resonance matches the exact response, for broad-banded earthquake ground motions, it would be preferable to evaluate it so that the areas under the frequency response curves of the two systems are apprnximately the same over a fairly wide range of frequencies extending to both sides of the resonant peak. This requires that the damping factor for the model be greater than the value defined by Eq. 59 .

For ground motions of the type recorded in the $1940 \mathrm{El} \mathrm{Centro} \mathrm{earthquake,} \mathrm{it} \mathrm{is} \mathrm{recommended} \mathrm{that} \mathrm{the}$ damping factor for the constant-parameter model be determined from

$$
\zeta^{*}=0.275+\frac{\delta}{2}
$$

and that its mass and stiffness be determined, as before. from Eqs. 57 and 58, respectively. For the recommended damping, the peak transmissibility for a system with $\delta=0.1$ turns out to be 1.9 , a value substantially lower than the exact value of 3.1 (see Fig. 4.3).

In Fig. 4.7, the exact response spectra for the maximum base shear in the wall of systems subjected to the El Centro earthquake record are compared with the corresponding spectra computed by the modified constant-parameter model. Two different values of the soil material damping factor. $\delta$. are considered. It is observed that, in both cases, the agreement between the two sets of results is indeed quite reasonable over the full range of natural frequencies examined. 


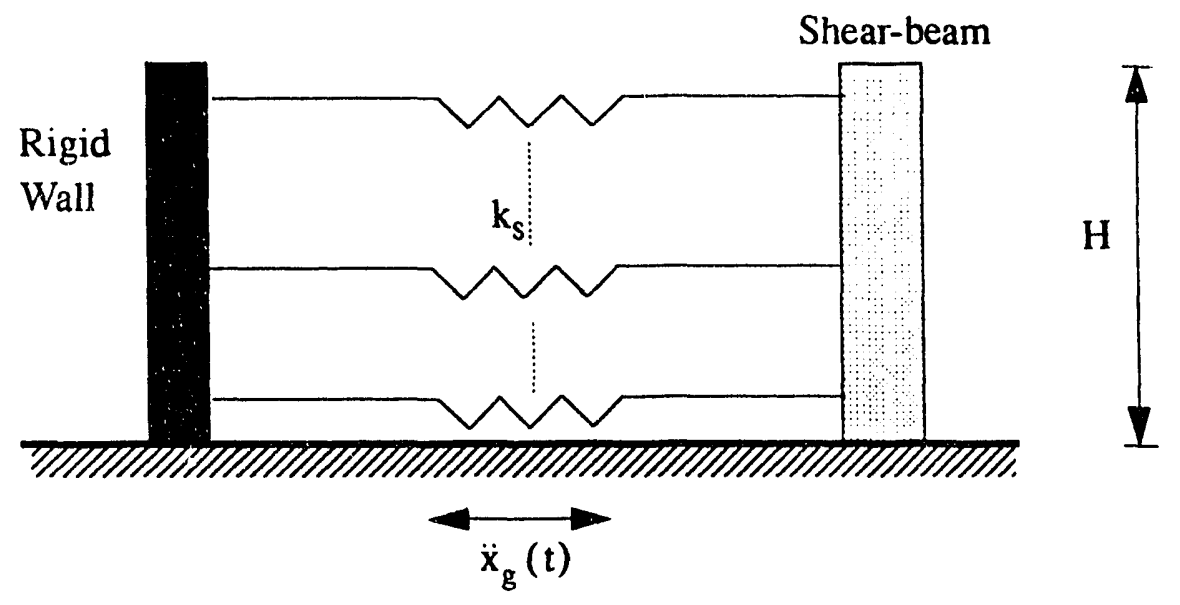

Figure 4.1 Scott's model. 

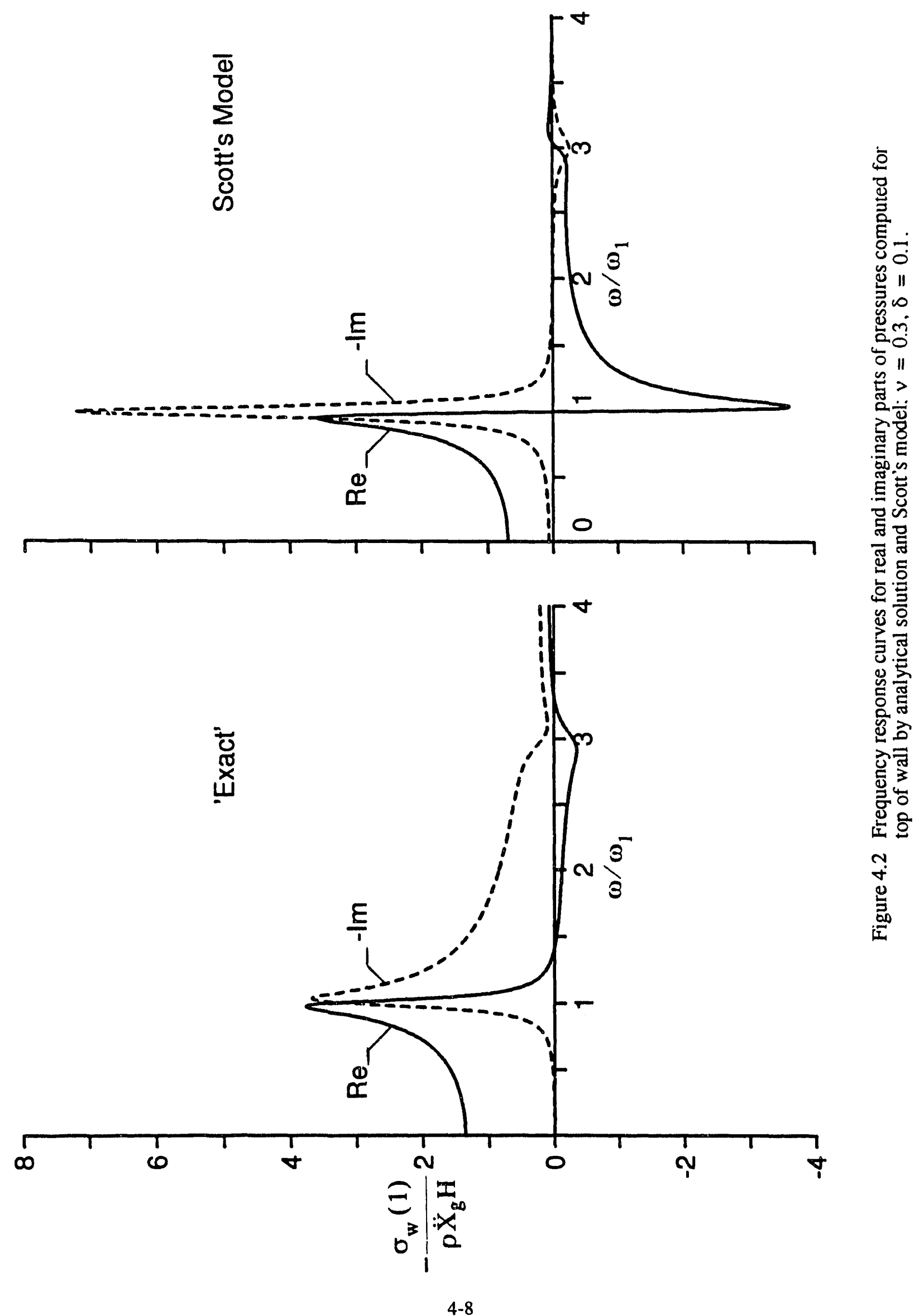


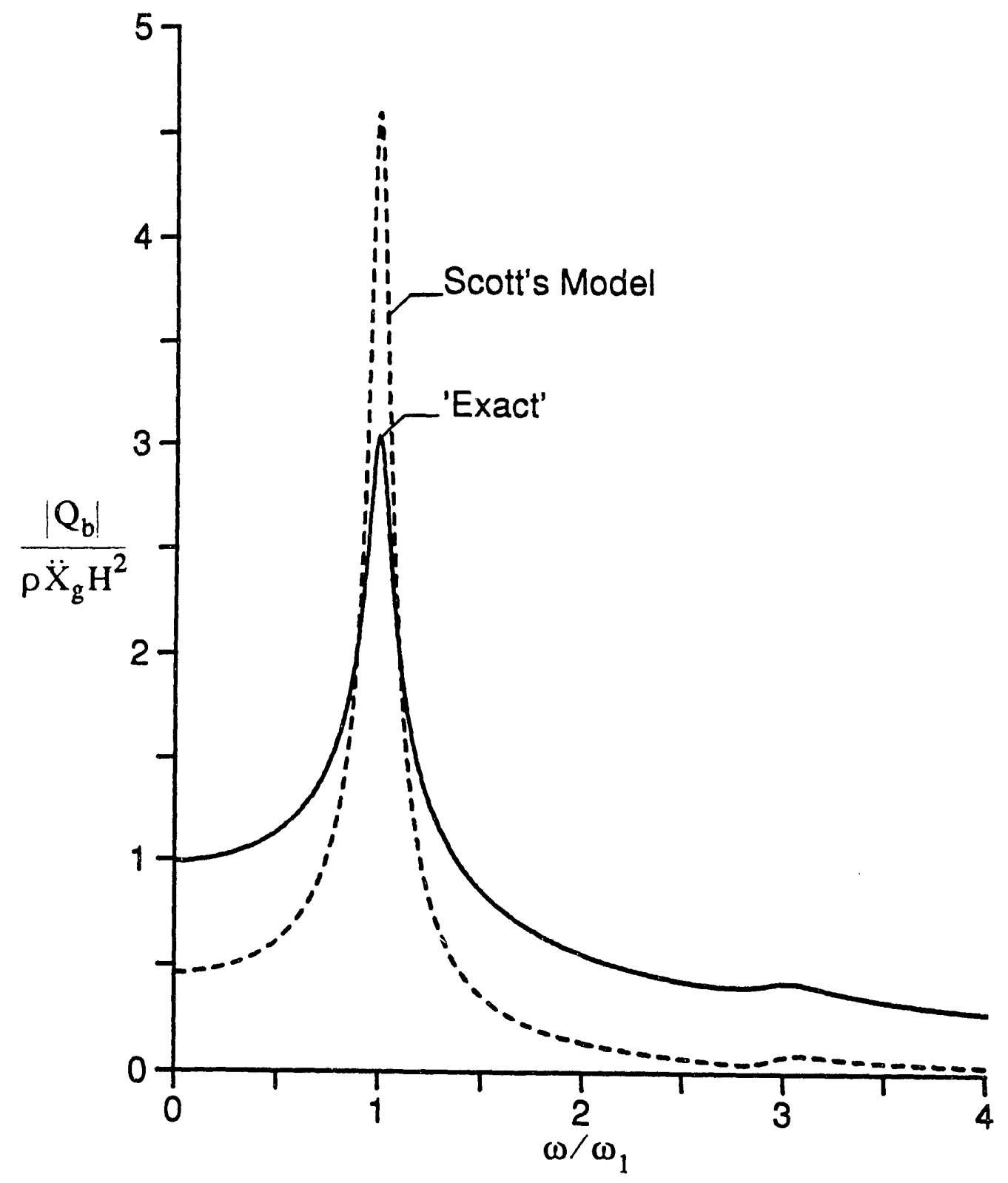

Figure 4.3 Comparison of frequency response curves for base wall-shears computed by analytical solution and Scott's model:

$v=0.3, \delta=0.1$. 


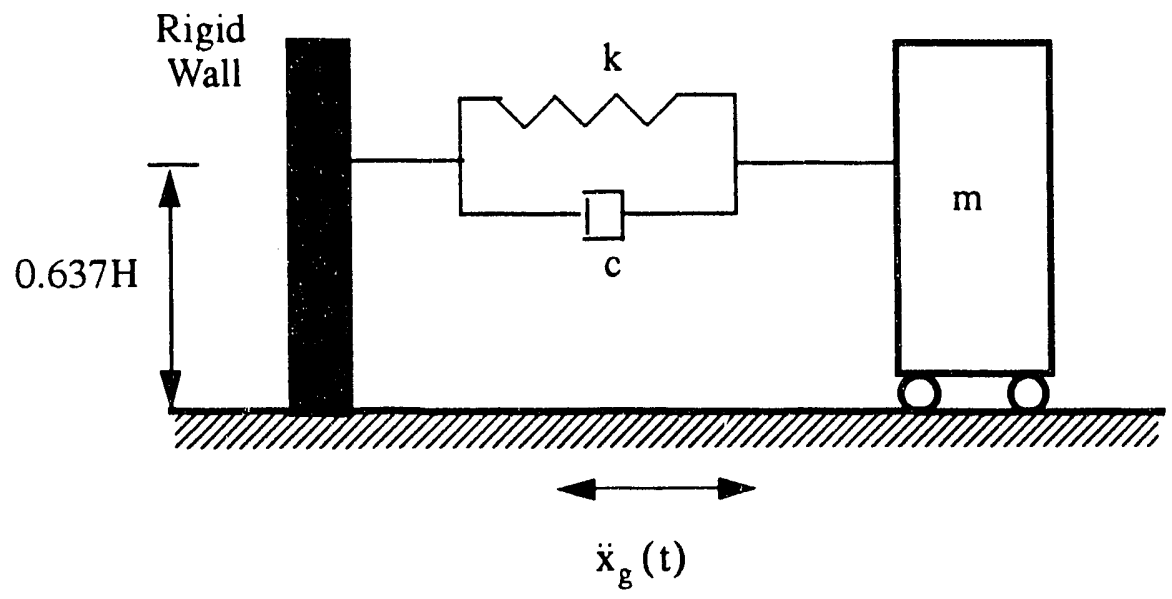

Figure 4.4 Proposed model. 


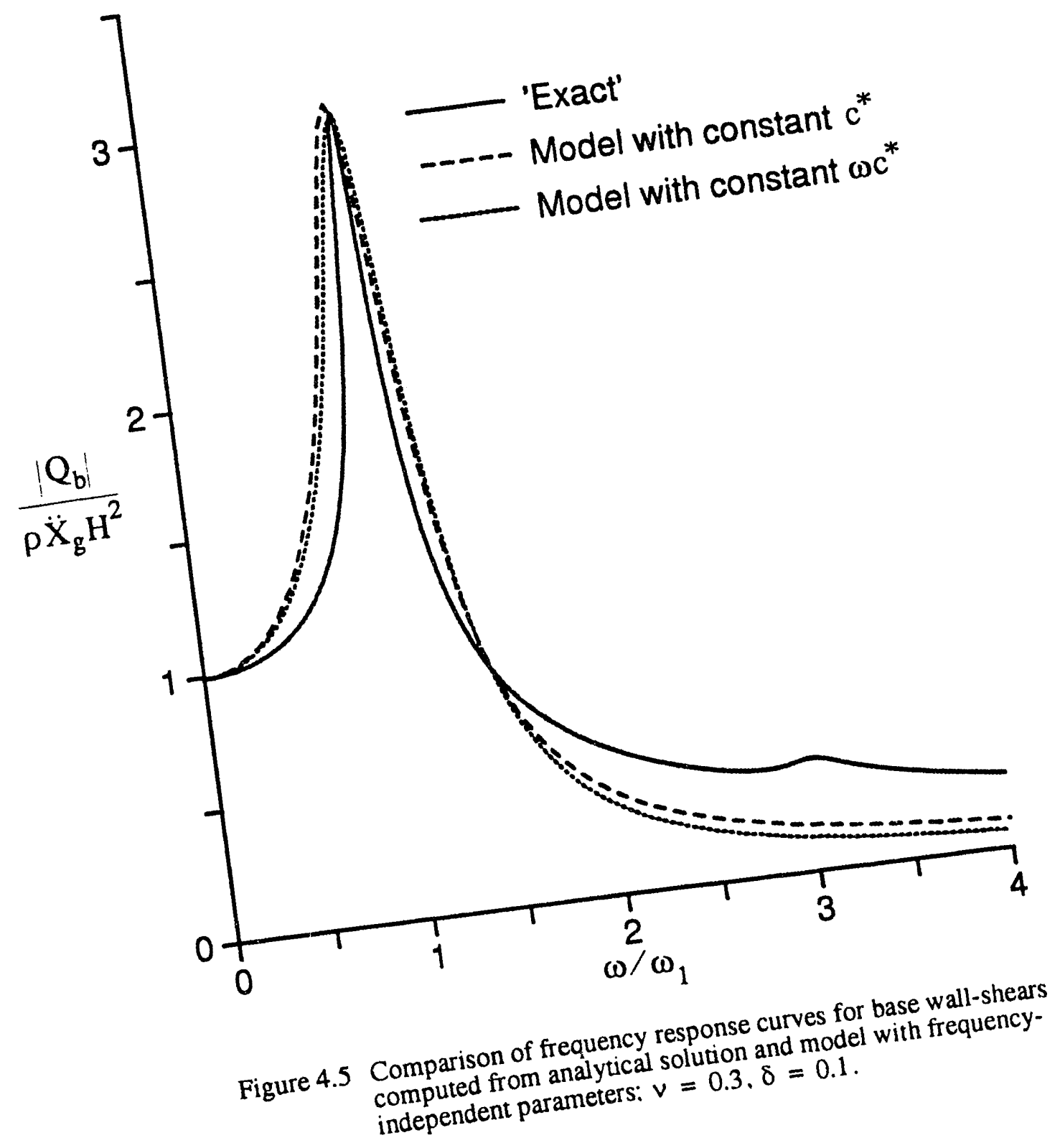




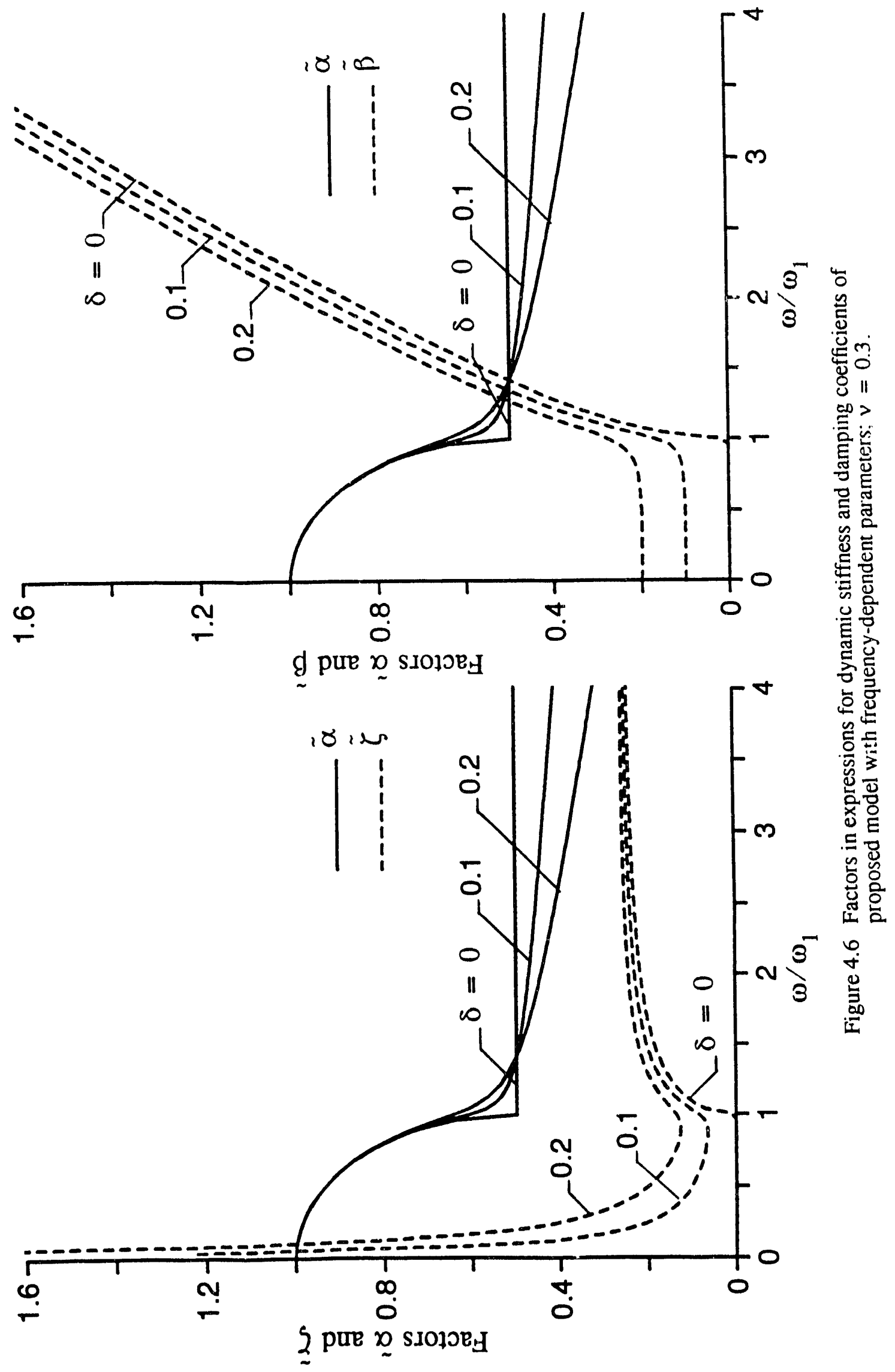




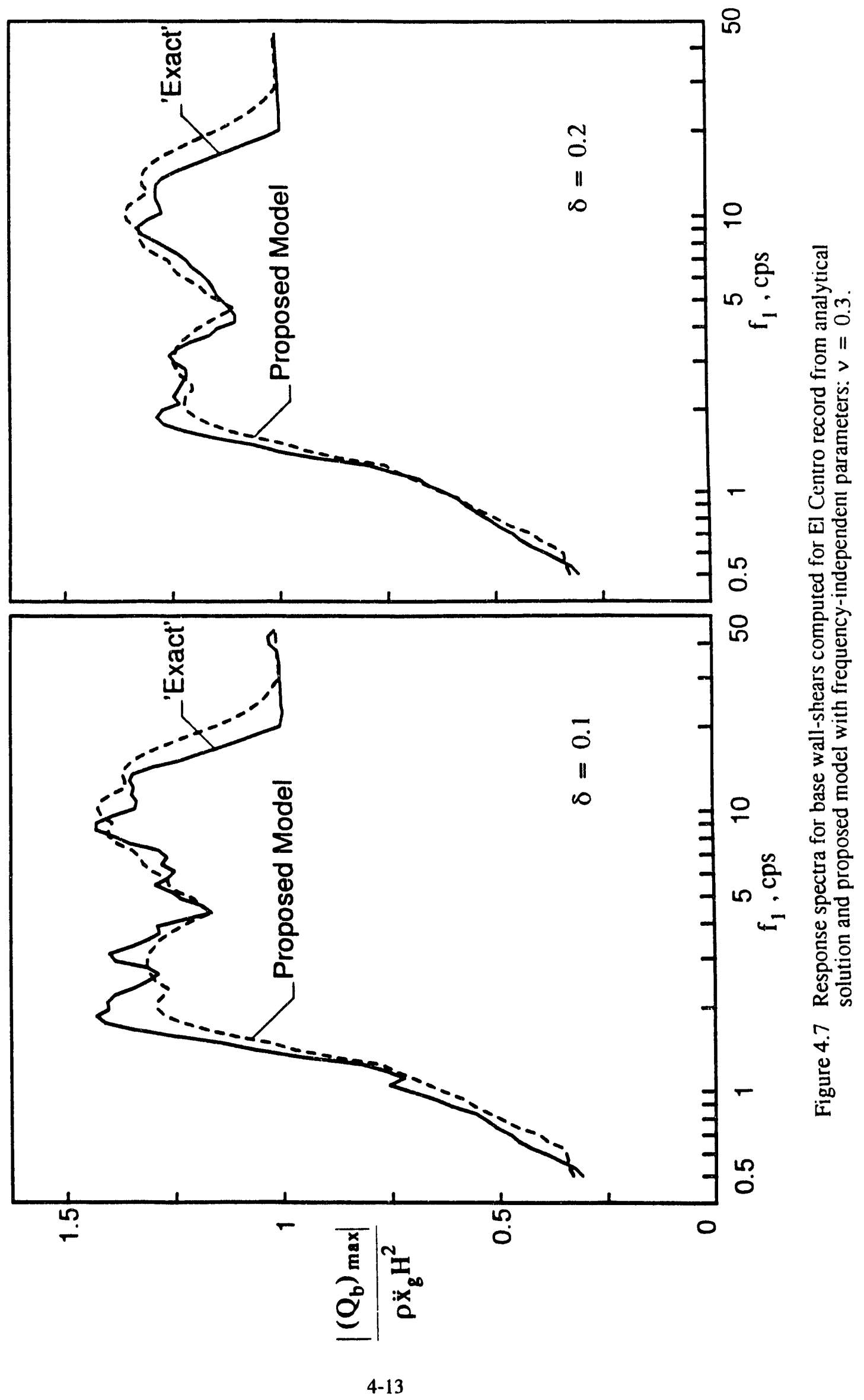




\section{SECTION 5}

\section{CONCLUSIONS}

The principal conclusions of this study may be summarized as follows:

1. With the analytical expressions developed herein, the dynamic pressures and associated forces induced by ground shaking on a vertical, rigid wall retaining a semi-infinite, uniform viscoelastic soil layer may be evaluated readily.

2. The comprehensive numerical data that have been presented and the analysis of these data provide valuable insights into the effects and relative importance of the numerous parameters involved, and a conceptual framework for the analysis and interpretation of the solutions for more involved systems as well.

3. Scott's model, which ignores the radiational damping capacity of the medium and considers the wall pressures to be proportional to the relative motions of the wall and the soil at the far field, does not adequately describe the action of the system and may lead to large errors.

4. The proposed alternative models, which effectively consider the wall pressures to be governed by the action of the soil in the immediate vicinity of the wall, better characterize the response of the system, and lead to results of reasonable accuracy. In particular, the model with frequency-independent parameters may be used to evaluate readily and reliably the effects of earthquake ground motions of broad-banded spectral characteristics 


\section{SECTION 6}

\section{REFERENCES}

1. H. N. Nazarian and A. H. Hadjian, 'Earthquake-induced lateral soil pressures on structures', $J$. geotech. eng. div. ASCE, 105. 1049-1066, (1979).

2. S. Prakash. 'Analysis of rigid retaining walls during earthquakes', Proc. Int. conf. on recent advances in geotech. earthquake eng. and soil dyn. St. Louis, Mo., 1981, III. pp. 1-28.

3. R. V. Whitman. 'Seismic design of earth retaining structures', Proc. 2nd Int. conf. on recent advances in geotech. earthquake eng. and soil dyn. St. Louis, Mo., 1991, II, pp. 1767-1778.

4. N. Mononobe and H. Matuo, 'On the determination of earth pressures during earthquakes', Proc. world eng. congress Tokyo, Japan, 1929, 9, paper No. 388.

5. S. Okabe. 'General theory of earth pressure and seismic stability of retaining wall and dam', $J$. Japan society' of civil engineers 12(1), (1924).

6. H. B. Seed and R. V. Whitman, 'Design of earth retaining structures for dynamic loads', ASCE speciality conf. on lateral stresses in the ground and design of earth retaining structures, 1970. pp. 103-147.

7. M. A. Sherif, I. Ishibashi and C. D. Lee, 'Earth pressure against rigid retaining walls', J. geotech. eng. div. ASCE. 108, 679-695, (1982).

8. M. A. Sherif and Y. F. Fang, 'Dynamic earth pressures on walls rotating about the top', Soils and Foundations 24(4). 109-117, (1984).

9. T. J. Siller, P. P. Christiano and J. Bielak, 'Seismic response of tied-back retaining walls', Earthquake eng. struct. dyn. 20, 605-620, (1991).

10. H. Matuo and S. Ohara. 'Lateral earth pressure and stability of quay walls during earthquakes', Proc. 2nd world conf. earthquake eng. Tokyo, Japan, (1960).

11. J. H. Wood, 'Earthquake-induced soil pressures on structures', Earthquake Engineering Rese، rch Laboratory, California Institue of Technology, Report EERL 73-05, 1973.

12. J. H. Wood, 'Earthquake-induced pressures on rigid wall structure', Bull. of New Zealand soc. for' earthquake eng. 8(3), 175-186, (1975).

13. R. F. Scott. 'Earthquake-induced pressures on retaining walls', Proc. 5th w'orld conf. on earthquake eng. Rome, Italy, II, 1611-1620, (1973).

14. S. Karkanias, 'Seismic behavior and simplified analysis of anchored sheet pile bulkheads', M.S. Thesis, Rensselaer Polytechnic Institute, Troy, N.Y., 1983.

15. K. T. Dennehy, " Seismic vulnerability, analysis and design of anchored bulkheads.' Ph.D. The- 
sis, Rensselaer Polytechnic Institute, Troy, N.Y., 1984.

16. S. K. Jain and R. F. Scott, 'Seismic analysis of cantilever retaining walls,' Proc. of SMIRT, Anaheim. Calif., 1989, pp. 241-246.

17. S. Alampalli and A. W. Elgamal, 'Retaining walls: computation of seismically induced deformations', Proc. 2nd Int. conf. on recent advances in geotech. earthquake eng. and soil dyn. St. Louis, Mo., 1991, 1. pp. 635-642.

18. C. Soydemir, 'Seismic design of rigid underground walls in New England', Proc. 2nd Int. conf. on recent advances in geotech. earthquake eng. and soil dyn. St. Louis, Mo., 1991, I, pp. 613620.

19. R. Flores-Berrones and R. V. Whitman, 'Seismic response of end-bearing piles', J. geotech. eng. div. ASCE, 108, 554-569, (1982).

20. C. A. Miller , C. J. Costantino and E. Heymsfeld. 'Soil-structure interaction effects on high level waste tanks', Proc. Third DOE natural phenomena hazards mitigation conf. St. Louis, Missouri, 1991, pp. 588-595.

21. A. S. Veletsos and B. Verbic, 'Vibration of viscoelastic foundations', Earthquake eng. struct. dyn. 2, 87-102, (1973).

22. A. S. Veletsos and K. W. Dotson. 'Horizontal impedances for radially inhomogeneous viscoelastic soil layers', Earthquake eng. struct. dyn. 16. 947-966, (1988).

23. H. Tajimi, 'Dynamic analysis of a structure embedded in an elastic stratum', Proc. 4th world conf. earthquake eng. Santiago, Chile, III(A-6), 53-69, (1969).

24. J. W. Cooley and J. W. Tukey, 'An algorithm for the machine calculation of complex Fourier series', Mathematics of Computation 19, 297-301, (1965).

25. A. S. Veletsos and C. E. Ventura. 'Dynamic analysis of structures by the DFT method'.J. struct. div. ASCE, 111, 2625-2642, (1985).

26. A. S. Veletsos and Y. Tang, 'Deterministic assessment of effecis of ground-motion incoherence', J. eng. mech. div. ASCE, 116, 1109-1124, (1990).

27. J. W. Meek, Personal commumication, 1992.

28. J. P. Wolf, Dynamic soil-structure interaction. Prentice-Hall, Englewoods Cliffs, N.J., 1985, pp. 114-127. 


\section{SECTION 7 \\ NOTATION}

The following symbols are used in this report:

\begin{tabular}{|c|c|}
\hline$A_{n}, B_{n}$ & constants of integration \\
\hline$a_{n}, b_{n}, c_{n}$ & real-valued dimensionless factors \\
\hline$\tilde{\mathbf{c}}$ & coefficient of viscous damping for model with frequency-dependent parameters \\
\hline$d_{n}$ & real-valued dimensionless factor \\
\hline$f_{1}$ & fundamental cyclic natural frequency of soil layer \\
\hline G & shear modulus of elasticity of soil material \\
\hline $\mathrm{G}^{*}$ & complex-valued shear modulus for soil material, defined by Eq. 6 \\
\hline$g_{1} \cdot g_{2}$ & dimensionsless factors in expression for dynamic wall pressure \\
\hline $\mathrm{H}$ & height of wall and soil layer \\
\hline$i$ & $\sqrt{-1}$ \\
\hline$\tilde{K}(\omega)$ & $\begin{array}{l}\text { complex-valued stiffness or impedance of model with frequency-dependent } \\
\text { characteristic }\end{array}$ \\
\hline$\tilde{\mathbf{k}}(\omega)$ & stiffness of spring for model with frequency-dependent parameters \\
\hline $\mathbf{k}^{*}$ & stiffness of spring for model with frequency-independent. constant properties \\
\hline $\begin{array}{l}\mathbf{k}_{\mathbf{s}} \\
\tilde{\mathbf{k}}_{\mathrm{st}}\end{array}$ & $\begin{array}{l}\text { stiffness of distributed springs in Scott's model } \\
\text { static values of } \tilde{K}(\omega) \text { and } \tilde{k}(\omega)\end{array}$ \\
\hline $\mathbf{M}_{\mathrm{b}}$ & bending moment at wall base \\
\hline $\mathbf{M}_{\mathrm{b}}^{\mathrm{st}}$ & static value of $M_{b}$ \\
\hline $\mathrm{m}^{*}$ & mass of model with frequency-independent, constant properties \\
\hline$\tilde{\mathrm{m}}$ & mass of model with frequency-dependent parameters; it is taken equal to $\mathrm{m}^{*}$ \\
\hline $\mathbf{n}$ & odd integer \\
\hline $\mathrm{Q}_{\mathrm{b}}$ & base shear in wall \\
\hline$Q_{b}^{\text {st }}$ & static value of $Q_{b}$ \\
\hline TR & $\begin{array}{l}\text { complex-valued transmissibility of system, representing ratio of actual base } \\
\text { shear induced in the wall to corresponding static value }\end{array}$ \\
\hline $\mathrm{TR}_{1}$ & value of $T R$ at $\omega=\omega_{1}$ \\
\hline$(\mathrm{TR})_{\mathrm{m}}$ & $\begin{array}{l}\text { complex-valued transmissibility of model, representing ratio of total force } \\
\text { transmitted to base of the model to the corresponding force transmitted under } \\
\text { static conditions of loading }\end{array}$ \\
\hline
\end{tabular}




\begin{tabular}{|c|c|}
\hline$t$ & time \\
\hline $\mathrm{U}$ & $\begin{array}{l}\text { complex-valued amplitude of horizontal displacement relative to moving } \\
\text { boundary for an arbitrary point of the medium }\end{array}$ \\
\hline $\mathrm{U}_{\mathrm{n}}$ & nth term in an expansion of $U$ \\
\hline $\mathrm{U}_{\mathrm{n}}^{\mathrm{h}}, \mathrm{U}_{\mathrm{n}}^{\mathrm{p}}$ & homogeneous and particular solutions of differential equation governing $U_{n}$ \\
\hline $\mathbf{u}$ & $\begin{array}{l}\text { horizontal displacement relative to the moving boundary of an arbitrary point of } \\
\text { the medium }\end{array}$ \\
\hline V & complex-valued amplitude of vertical displacement of a point of the medium \\
\hline $\mathrm{v}$ & vertical displacement of an arbitrary point of the medium \\
\hline$v_{\mathbf{s}}$ & velocity of shear-wave propagation of medium \\
\hline $\mathrm{X}_{\mathrm{g}}, \dot{\mathrm{X}}_{\mathrm{g}}, \ddot{\mathrm{X}}_{\mathrm{g}}$ & $\begin{array}{l}\text { amplitudes of harmonic ground displacement, velocity and acceleration, } \\
\text { respectively }\end{array}$ \\
\hline $\mathrm{x}$ & horizontal position coordinate \\
\hline$x_{g}, \dot{x}_{g}, \ddot{x}_{g}$ & $\begin{array}{l}\text { maximum values of displacement, velocity and acceleration of ground for a } \\
\text { transient ground shaking }\end{array}$ \\
\hline$\ddot{x}_{g}(t)$ & instantaneous value of ground acceleration \\
\hline $\mathrm{y}$ & vertical position coordinate \\
\hline$\alpha_{n}$ & complex-valued dimensionless factor \\
\hline$\tilde{\alpha}$ & dynamic stiffness coefficient for model with frequency-dependent parameters \\
\hline$\hat{\beta}$ & $\begin{array}{l}\text { dimensionsless damping coefficient for model with frequency-dependent } \\
\text { parameters }\end{array}$ \\
\hline$\delta$ & material damping factor for constant hysteretic characterization of soil damping \\
\hline$\zeta^{*}, \tilde{\zeta}$ & $\begin{array}{l}\text { damping factors, in percent of critical damping, for models with frequency } \\
\text { independent and frequency-dependent parameters, respectively }\end{array}$ \\
\hline$\eta$ & $\mathrm{y} / \mathrm{H}=$ dimensionless vertical position coordinate \\
\hline$\theta_{n}$ & phase angle defined by Eq. 28 \\
\hline$\Lambda_{\mathrm{n}}$ & dimensionless factor defined by Eq. 22 \\
\hline$\lambda^{*}$ & complex-valued Lame constant defined by Eq. 7 \\
\hline$v$ & Poisson`s ratio for soil \\
\hline$\xi$ & $\mathrm{x} / \mathrm{H}=$ dimensionless horizontal position coordinate \\
\hline$\rho$ & mass density for medium \\
\hline$\sigma$ & complex-valued amplitude of horizontal normal stress at an arbitrary point \\
\hline$\sigma_{w}$ & normal wall pressure \\
\hline$\sigma_{w}^{s t}$ & static value of $\sigma_{w}$ \\
\hline$\sigma_{\mathrm{x}}$ & horizontal normal stress at an arbitrary point and time \\
\hline$\tau$ & complex-valued amplitude of shearing stress at an arbitrary point \\
\hline$\tau_{x y}$ & shearing stress at an arbitrary point and time \\
\hline$\phi_{\mathrm{n}}$ & frequency ratio defined by Eq. 23 \\
\hline$\chi_{0}$ & function of $v$ defined by Eq. 16 \\
\hline
\end{tabular}


$\Psi_{e}, \Psi_{0} \quad$ functions of $v$ defined by Eqs. 12 and 10, respectively

$\Psi_{v}, \Psi_{\sigma} \quad$ functions of $v$ defined by Eqs. 36 and 40 , respectively

$\omega$ circular frequency of excitation and resulting steady-state motion

$\omega_{1}$ fundamental circular natural frequency of stratum cosidered to act as a series of vertical cantilever shear-beams 


\section{SECTION 8}

\section{APPENDIX}

\subsection{Harmonic Response of Uniform Shear-Beam}

The response of a base-excited, uniform shear-beam with constant hysteretic damping is governed by the differential equation

$$
\frac{G^{*}}{H^{2}} \frac{\partial^{2} u}{\partial \eta^{2}}=\rho \frac{\partial^{2} u}{\partial t^{2}}+\rho \ddot{x}_{g}(t)
$$

in which $\ddot{x}_{\mathrm{g}}(t)=$ the base acceleration at any time $t ; u=$ the displacement of the beam relative to the moving base: $H=$ the height of the beam; $\eta=y / H=$ the dimensionless position coordinate for a point of the beam at a distance $y$ from the base; $\rho=$ the mass density of the beam; $G^{*}=$ its complex-valued shear modulus defined by

$$
G^{*}=G(1+i \delta)
$$

$\mathrm{G}=$ the real-valued modulus; and $\delta=$ the constant hysteretic material damping factor, frequently denoted as $2 \beta$ or $2 \bar{\xi}$ by others.

For a harmonic base acceleration.

$$
\ddot{x}_{g}(t)=\ddot{X}_{g} e^{i \omega t}
$$

in which $\ddot{X}_{g}=$ the amplitude of the acceleration; $\omega=$ its circular frequency, and $i=\sqrt{-1}$. The beam displacement may be expressed as

$$
u(\eta, t)=U(\eta) e^{i \omega t}
$$

in which $U(\eta)$ is a function of $\eta$ only.

On substituting Eq. A.4 into Eq. A.1 and cancelling the common exponential function, one obtains the ordinary differential equation

$$
\frac{G^{*}}{\rho H^{2}} \frac{d^{2} U}{d \eta^{2}}+\omega^{2} U=\ddot{X}_{g}
$$

The solution of Eq. A.5 may be taken in the form 


$$
U(\eta)=\sum_{n=1,3}^{\infty} C_{n} \sin \frac{n \pi}{2} \eta
$$

in which $C_{n}$ are constants to be determined, and each term of the series satisfies the condition of $\mathrm{U}=0$ at $\eta=0$ and $\mathrm{U}^{\prime}=0$ at $\eta=1$; a prime superscript denotes differentiation with respect to $\eta$. Next, the constant $\ddot{X}_{\mathrm{g}}$ is expressed in the form

$$
\ddot{X}_{g}=\frac{4}{\pi} \ddot{X}_{g} \sum_{n=1,3}^{\infty} \frac{1}{n} \sin \frac{n \pi}{2} \eta
$$

and Eqs. A.6 and A.7 are substituted into Eq. A.5 to obtain

$$
C_{n}=-\frac{16}{\pi^{3}} \frac{\rho \ddot{X}_{g} H^{2}}{G} \frac{1}{n^{3}} \frac{1}{1-\phi_{n}^{2}+i \delta}
$$

in which

$$
\begin{aligned}
& \phi_{\mathrm{n}}=\frac{1}{\mathrm{n}} \frac{\omega}{\omega_{1}} \\
& \omega_{1}=\frac{\pi v_{s}}{2} \overline{\mathrm{H}}
\end{aligned}
$$

and $v_{s}=$ the shear wave velocity for the material of the beam, defined by

$$
v_{s}=\sqrt{\frac{G}{\rho}}
$$

The resulting expression for the instantaneous value of the relative displacement, $u(\eta, t)$, is

$$
u(\eta, t)=-\frac{16}{\pi^{3}} \frac{\rho \ddot{X}_{g} H^{2}}{G}\left[\sum_{n=1,3}^{\infty} \frac{1}{n^{3}} \frac{1}{1-\phi_{n}^{2}+i \delta} \sin \frac{n \pi}{2} \eta\right] e^{i \omega t}
$$



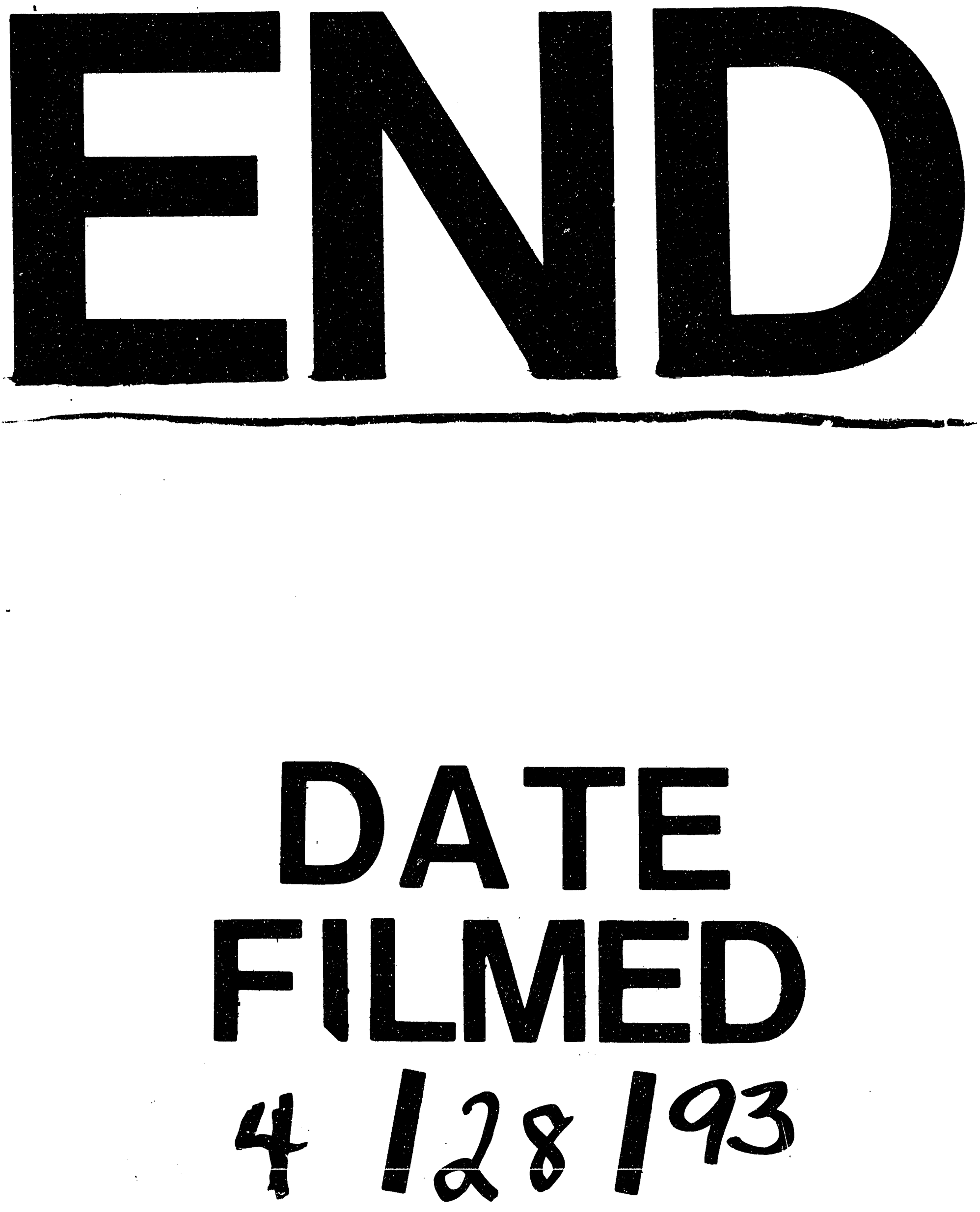
